\title{
Targeting Noradrenergic Neurons of the Locus Coeruleus: A Comparison of Model Systems and Strategies
}

\author{
Chantal Wissing ${ }^{1}$, Maxime Maheu $^{1,2}$, Simon Wiegert $^{1 *}$, Alexander Dieter $^{1}$ \\ ${ }^{1}$ Synaptic Wiring Lab, Center for Molecular Neurobiology, University \\ Medical Center Hamburg-Eppendorf, Hamburg, Germany \\ ${ }^{2}$ Section Computational Cognitive Neuroscience, Department of Neurophysiology and \\ Pathophysiology, University Medical Center Hamburg-Eppendorf, Hamburg, Germany \\ *Correspondence: simon.wiegert@zmnh.uni-hamburg.de
}

\begin{abstract}
The locus coeruleus (LC) noradrenergic system is involved in a plethora of physiological and pathophysiological processes. Refining our understanding of LC function largely relies on selective transgene expression in molecularly defined cells, enabling targeted manipulation and read-out of noradrenergic neurons. Here, we performed a side-by-side comparison of the most commonly used strategies and model systems enabling genetic access to the locus coeruleus. We report substantial differences among them both in terms of transgene expression efficacy, and in their molecular specificity. These findings are of critical importance for interpreting the results obtained from past experiments using the respective targeting strategies, as well as for the design of future studies.
\end{abstract}

\section{Introduction}

The locus coeruleus (LC) - a bilateral nucleus located in the brainstem - is the main source of noradrenaline (or norepinephrine; NE) in the central nervous system. Despite its small size $\left(\sim 3,000\right.$ neurons in the rodent brain $\left.^{1}\right)$, the LC is involved in various physiological functions, ranging from the modulation of brain states such as alertness and arousal, over sensory perception and processing, up to attention, learning, and memory consolidation ${ }^{2-4}$. Furthermore, the LC has been linked to numerous pathological conditions, including Alzheimer's and Parkinson's disease, depression, and post-traumatic stress disorder ${ }^{2-6}$.

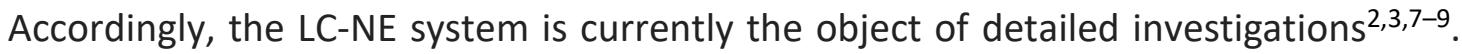
Recent research points towards a high modularity of this small nucleus with subpopulations of cells targeting different brain regions putatively playing different physiological roles ${ }^{2}$. Thus, the LC might exert more refined, target-specific modulation of brain activity than previously expected. These insights brought the LC-NE system to the focus of many research groups, which rely on specific targeting of LC-NE neurons to interrogate LC function ${ }^{2}$. Key technologies to access the LC-NE system include genetic tools such as cre driver lines and viral transduction, which enable access to molecularly identified neural populations, even in a projection-specific 
manner. Genetic access to the LC gained increasing relevance with the development of novel reporter tools, such as genetically encoded activity sensors for noradrenaline ${ }^{10,11}$ and dopamine ${ }^{12-14}$, or when implementing manipulations of LC subpopulations in a projectionspecific manner with opto- or chemogenetic tools. Genetic access to the LC-NE system can be achieved with different strategies, including transgenic driver lines (Supplementary table S1), and promoter-driven transgene expression. Driver lines express cre recombinase under control of the tyrosine hydroxylase $(\mathrm{TH})$ promoter both in mice ${ }^{15-17}$ and rats ${ }^{18}$, as well as the dopamine- $\beta$-hydroxylase promoter $(\mathrm{DBH})^{16,19}$ and the promoter for a norepinephrine transporter (NET) in mice ${ }^{20}$. TH is the enzyme involved in the synthesis of L-DOPA from tyrosine, which occurs three reaction steps upstream of the final product NE and therefore occurs in neurons that produce dopamine (DA) and/or NE. DBH catalyzes the synthesis of NE from DA and is therefore specific for NE-neurons. Similarly, NET, the membrane protein mediating reuptake of extracellular NE, is used as a selective marker for NE-releasing neurons.

Alternative approaches to target the LC-NE system which do not rely on transgenic lines have also been proposed. Notably, NE-specific transgene expression can be achieved using a minimal synthetic promoter, which contains multiple copies of a cis regulatory element (Phox2a/Phox2b response site; PRS) derived from the human DBH promoter. A synthetic promoter, composed of 8 PRS copies $\left(\mathrm{PRS} \times 8^{21}\right)$, was shown to specifically transduce noradrenergic cells both in mice ${ }^{22,23}$ and in rats ${ }^{21,24}$. While the availability of these four strategies $(T H-, D B H-, N E T$-cre, and PRS $\times 8)$ for precise targeting of the LC-NE-system provides a wide range of alternatives to choose from, many systems for genetic manipulations come with different caveats and challenges (for reviews, see e.g. ${ }^{25-27}$ ). Crucially, a direct comparison of the strategies to target the LC noradrenergic system is still lacking, and proper quantification of viral transduction is often not reported.

Here, we performed a side-by-side comparison of virus-mediated transgene expression in the noradrenergic system by introducing conditional, cre-dependent reporter constructs into the LC of the commercially available $\mathrm{TH}$ - and $\mathrm{DBH}$-transgenic mice, and unconditional reporter constructs under control of the PRS $\times 8$-promoter into the LC of wildtype mice. We report substantial differences in the patterns of transgene expression at the cellular level, both in terms of efficacy and molecular specificity. These findings are of great importance to the growing community of researchers implementing genetic manipulations of the noradrenergic system to investigate LC function. Notably, our findings might guide the choice for an adequate targeting strategy in future research, and should be considered when interpreting the data obtained from the respective model system. Furthermore, our results highlight the need for detailed verification of transgenic model systems in neuroscientific research in general. 


\section{Results}

\section{Efficacy and selectivity of transgene expression}

In order to visualize transgene expression, we bilaterally injected the locus coeruleus with titer-matched suspensions of recombinant adeno-associated virus (pseudotype 2/9; rAAV2/9) carrying constructs encoding the enhanced green fluorescent protein (eGFP; Fig. 1a). In $D B H-$ and $T H$-transgenic mouse lines, gene expression was controlled by combining double-floxed inverted open reading frames $(\mathrm{DIO})^{28}$ of the transgene with a strong, synthetic promotor $(\mathrm{CAG})^{29}$ allowing for conditional gene expression in a cre-dependent manner. In wild-type mice (C57BL/6), the reporter gene was expressed under control of the synthetic PRS $\times 8$ promoter. Six weeks after injection, we analyzed transgene expression by confocal microscopy of coronal sections which were immuno-stained against TH (to visualize noradrenergic neurons in the LC) and GFP (to enhance the signal originating from transgene expression; Fig. 1b).

To quantify transgene expression on a cellular level, we used the recently developed deep-learning-based algorithm CellPose ${ }^{30}$ to segment cells expressing $\mathrm{TH}\left(\mathrm{TH}^{+}\right)$or eGFP $\left(\mathrm{eGFP}^{+}\right.$), respectively (Fig. 1c; only LC in which transgene expression was observed were included). Segmentation was done separately for each color channel, resulting in a total of 3266/2937, 2922/2582, and 2861/3430 $\mathrm{TH}^{+} / \mathrm{eGFP}{ }^{+}$cells in $\mathrm{DBH}-, \mathrm{TH}-$, and PRS $\times 8$-animals respectively (33/31/30 brain slices; $11 / 11 / 10 \mathrm{LC} ; n=7 / 7 / 7$ mice). The average number of $\mathrm{TH}^{+}$ cells per analyzed slice was comparable across approaches, with $98.9 \pm 24.7,94.3 \pm 15.1$, $95.4 \pm 15.0$ cells (mean \pm standard deviation, $S D$ ) for $D B H, T H$, and PRS $\times 8$, respectively.

To evaluate the efficacy of viral transduction, defined as the fraction of $\mathrm{TH}^{+}$cells coexpressing eGFP over the total number of $\mathrm{TH}^{+}$cells, we compared the overlap between the segmented masks of each $\mathrm{TH}^{+}$cell with the masks of eGFP+ cells, defining cells with an overlap of $\geq 50 \%$ of the $\mathrm{TH}^{+}$cell mask as positively transduced. Similarly, we quantified the specificity of transgene expression as the fraction of $\mathrm{eGFP}^{+}$cells that co-express $\mathrm{TH}$ over the total number of eGFP ${ }^{+}$cells. Doing so, we found substantial differences both in efficacy (one-way ANOVA, $F_{18}=12.7, p=0.0004$ ) and in specificity (one-way ANOVA, $F_{18}=32.7, p=1 \times 10^{-6}$ ) between model systems (Fig. 1d): while no difference was detected between the efficacy of $D B H$ - and PRS $\times 8$-mediated transgene expression (74.3 $\pm 10.5 \%$ vs. $77.7 \pm 4.8 \%$; mean \pm SD; Tukey's test, $p=0.88)$, the efficacy of $T H$-mediated expression was significantly lower $(44.9 \pm 20.1 \%$ mean \pm SD; Tukey's test, $p=0.0018 / 0.0006$, compared against $D B H / P R S \times 8$, respectively; Fig. 1d, $y$-axis).

In terms of molecular specificity, differences were observed between all three approaches, with the highest specificity achieved by $D B H$-cre $(80.1 \pm 9.0 \%$; Tukey's test against PRS $\times 8 /$ TH-cre, $\left.p=0.0012 / 6.1 \times 10^{-7}\right)$, followed by PRS $\times 8-(62.2 \pm 3.9 \%$; Tukey's test against TH-cre, $p=0.0038$ ) and $T H$-mediated gene expression (46.6 $\pm 9.2 \%$; Fig. 1d, $\mathbf{x}$-axis). 
bioRxiv preprint doi: https://doi.org/10.1101/2022.01.22.477348; this version posted January 23, 2022. The copyright holder for this preprint (which was not certified by peer review) is the author/funder. All rights reserved. No reuse allowed without permission.

a

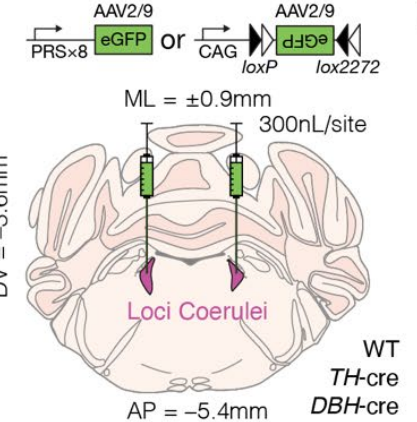

C
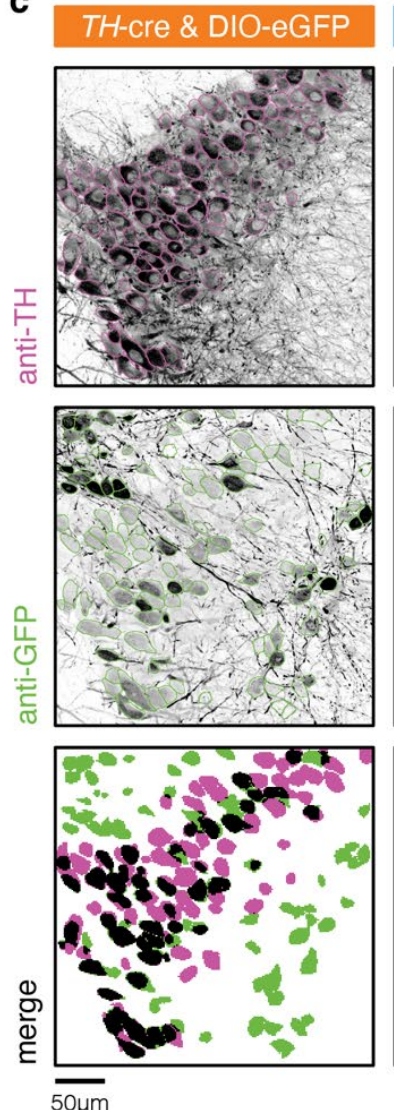

d

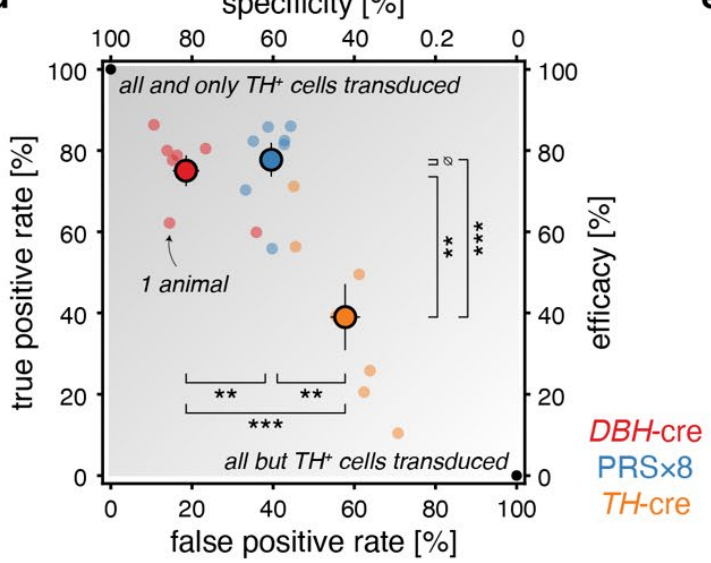

b Immuno-staining of example coronal slice
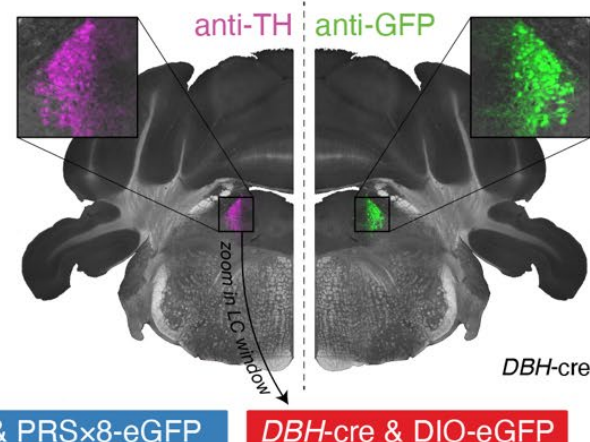

WT \& PRS $\times 8$-eGFP
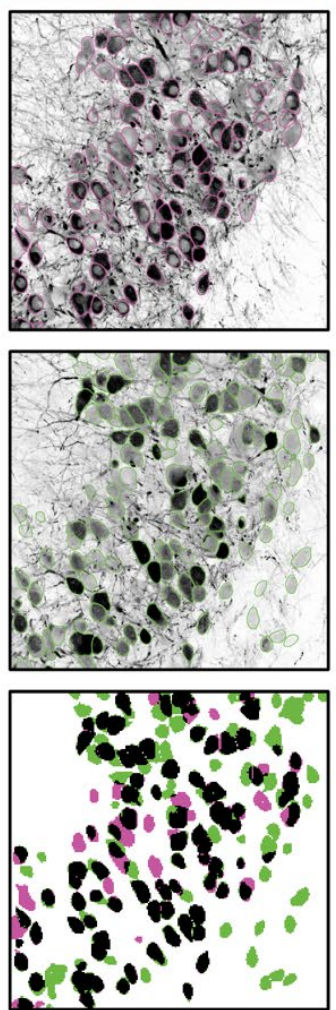

$\mathrm{TH}^{+}$cell $\mathrm{GFP}^{+}$cell

intersection
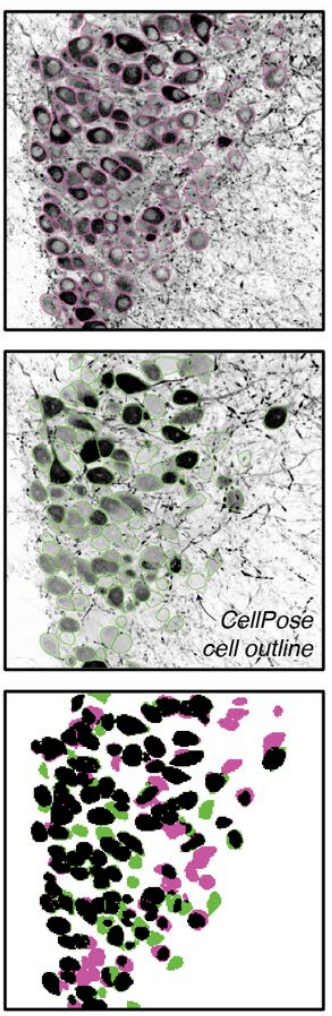

e

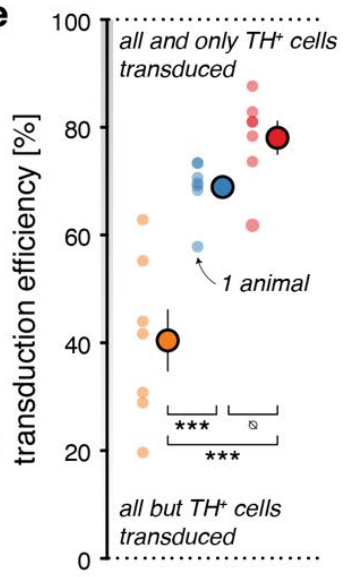

Figure 1 | Viral transduction of locus coeruleus noradrenergic neurons in different model systems. a, Either PRS $\times 8$-eGFP (in WT) or CAG-DIO-eGFP (in $\mathrm{TH}$ - and $D B H$-cre) was bilaterally injected into the LC of mice $(n=7$ animals in each group). Brain slice image from ${ }^{31} \mathbf{b}$, Example LC hemi-brain slice (vertically mirrored) from a $D B H$-cre animal. Noradrenergic neurons were identified by immunostaining against TH (left, magenta), while transgene expression was visualized with an anti-GFP staining (right, green). c, Example LC cells in each of the three model systems (columns) immuno-stained against TH (first row, magenta outlines) and GFP (second row, green outlines). Cells were identified with CellPose and boundaries are denoted with colored outlines. Resulting cell masks were then overlaid (third row), and each $\mathrm{TH}^{+}$cell was labeled as GFP' (successful transduction, black) when cell masks overlapped $50 \%$ or more the size of the $\mathrm{TH}^{+}$cell, or GFPotherwise (missed transduction, magenta). $\mathrm{GFP}^{+}$and $\mathrm{TH}^{-}$ (erroneous transduction, green) was counted as unspecific transduction. d, From cell counts, two quantities were computed for each model system: On the one hand, the true positive rate (yaxis) is computed as $N\left(\mathrm{GFP}^{+} \mid \mathrm{TH}^{+}\right) / N\left(\mathrm{TH}^{+}\right)$and reflects efficacy of $\mathrm{TH}^{+}$cell transduction. On the other hand, specificity (the inverse of the false positive rate, $x$-axis) is computed as $N\left(\mathrm{TH}^{+} \mid \mathrm{GFP}^{+}\right) / N\left(\mathrm{GFP}^{+}\right) \quad$ and reflects how specific one approach is in transducing exclusively $\mathrm{TH}^{+}$cells (while ignoring $\mathrm{TH}^{-}$cells). $D B H$-cre and PRS $\times 8$ approaches have similar efficacy, which is greater than $\mathrm{TH}$ cre. Specificity is largest in $D B H$ cre, followed by PRS $\times 8$, and $T H$ cre. e, Efficacy and specificity were combined into a single metric summarizing the transduction efficiency. This metric formally corresponds to taking the area under the (1-point) ROC curve (in d)

(specificity + efficacy) / 2. DBH-cre and $P R S \times 8$ show similar efficiency, while both are more efficient than $\mathrm{TH}$-cre. In $\mathbf{d}$ and e, error bars denote the standard error of the mean (with $n=7$ ), while statistical significance is denoted by $\varnothing$ for non-significance, and $* * * / * * *$ for $p<0.05 / 0.01 / 0.001$, respectively. 
We ultimately summarized efficacy and specificity into a single metric termed "transduction efficiency" (formally equivalent to the area under the ROC curve, or the average of efficacy and specificity). Transduction efficiency was different across groups (one-way ANOVA, $F_{18}=24.5, p=7.2 \times 10^{-6}$; Fig. 1e): $D B H$-cre and PRS $\times 8$ induced a more efficient transduction than $\mathrm{TH}$-cre (difference to $\mathrm{DBH}$-cre $=37.6 \% \pm 12.7 \%$, Tukey's test, $p=7.7 \times 10^{-6}$; difference to PRS $\times 8=28.5 \% \pm 14.5 \%$, Tukey's test, $p=2.2 \times 10^{-4}$ ). However, DBH-cre was not found to be significantly more transduction efficient than PRS $\times 8$ (difference $=9.1 \% \pm 5.6 \%$, Tukey's test, $p=0.26)$.

\section{Controls for possible quantification biases}

As these conclusions depend on the overlap threshold chosen to define co-expression ( $\geq 50 \%$ ), we systematically varied the threshold from $5-95 \%$ (in $5 \%$ increments) and showed that relative differences of both efficacy and specificity were independent of the chosen overlap threshold (Fig. S1). Another factor potentially biasing our conclusions might be unspecific transgene expression due to leakage of the cre-dependent constructs injected in $\mathrm{DBH}$ - and $\mathrm{TH}$-cre animals ${ }^{32}$. To test this possibility, we injected the same virus in 4 LC of 2 wildtype mice. We observed lack of eGFP expressing cells in the absence of cre-recombinase, thereby ruling out a bias due to potential viral leakage (Fig. S2). Finally, to account for possible segmentation errors, we compared results obtained from CellPose with manual cell identification from two experienced independent experimenters ( $C W$ and $A D$ ) in a subset of slices. We found significant agreement between CellPose and experimenter rating, as well as between experimenters (Fig. S3), indicating that the results obtained with CellPose were robust.

In our experiments, brain slices were immunostained against GFP to increase fluorescence and facilitate detection of transduced cells. However, the anti-GFP-staining might have exaggerated the fluorescence of not meaningful, very weakly expressing, eGFP ${ }^{+}$ cells $^{33}$, and thereby artificially increased efficacy, while decreasing specificity (albeit uniformly across approaches). To examine the extent of this effect, we immunostained against GFP using a secondary antibody in the red spectrum, and directly compared cells detected in the green (native eGFP) and red (GFP-stained) channels in a subset of animals ( $n=3,6,4$ for $D B H-$, $\mathrm{PRS} \times 8, \mathrm{TH}$-cre respectively; Fig. 2a).

First, we measured whether anti-GFP immunostaining increased the number of fluorescent cells as detected by CellPose. Indeed, we found that $20.9 \pm 14.8 \%$ more cells were detected in the GFP-stained than in the native eGFP channel (absolute difference: $15.5 \pm 12.0$ cells; $t$-test against 0: $t_{12}=4.66, p=5.5 \times 10^{-4}$; Fig. 2 b). Second, in addition to the overall increase in the number of detected cells, we quantified the proportion of cells revealed by the anti-GFP immunostaining that could not be found in the native eGFP channel. We found that cells segmented in the anti-GFP channel failed to co-localize with $36.1 \pm 12.4 \%$ (overlap threshold of $\geq 50 \%$ ) of the eGFP ${ }^{+}$cells (Fig. 2c,d). Finally, we aimed to understand the changes in fluorescence induced by the anti-GFP immunostaining, in particular with respect to weakly 
expressing eGFP cells (Fig. 2e). To do so, we fitted a non-linear function (Fig. 2f) describing the fluorescence of GFP-stained cells as a function of the native eGFP fluorescence (after withinchannel normalization; Fig. 2e). The first parameter of this function quantifies the overall change in fluorescence induced by the immunostaining (Fig. 2f, top panel), which was found significantly larger than 0 (gain $=0.38 \pm 0.54$; $t$-test against $0: t_{12}=2.54, p=0.026$; Fig. $2 \mathrm{~g}$ ). The second parameter accounts for a possible asymmetry in the fluorescence increase depending on the native eGFP fluorescence (Fig. 2f, bottom panel). This parameter was significantly smaller than 1 (bias $=0.81 \pm 0.30 ; t$-test against $1: t_{12}=-2.30, p=0.04$ ), indicating that fluorescence increase was substantially stronger for the more weakly (dimmest) eGFPexpressing cells. Altogether, these results suggest that the anti-GFP immunostaining increased the fluorescence of weakly expressing eGFP cells, thereby causing an inflated number of GFP ${ }^{+}$ cells detected by CellPose, most likely translating into larger apparent efficacy and lower apparent specificity values than what the native eGFP expression would have given. Noteworthy, this effect similarly applies to all across groups (Fig. 2), and the functional relevance of those weakly transduced cells remains open for future research.
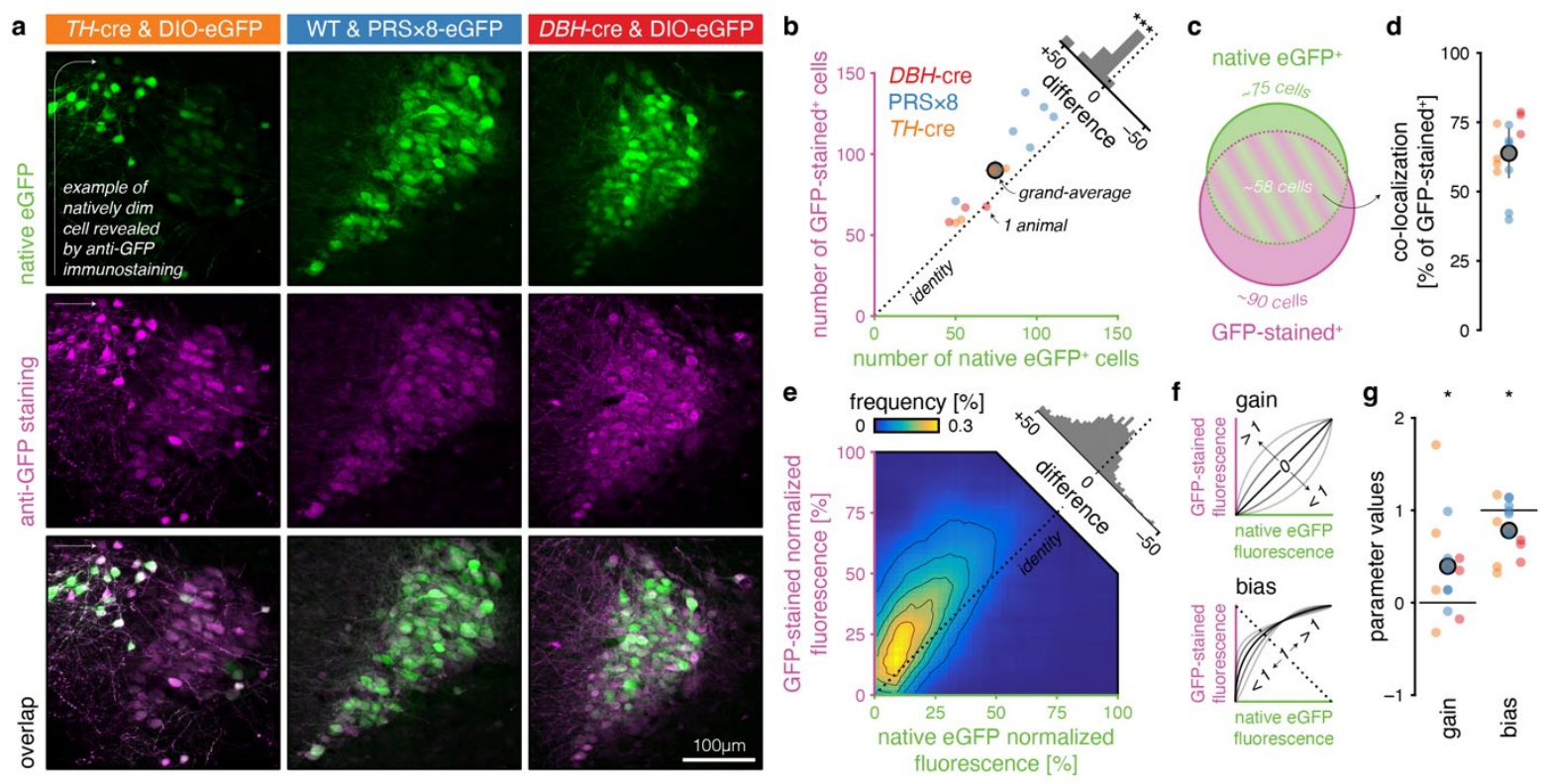

Figure 2 | Comparison of native eGFP expression with anti-GFP staining. a, Example brain slices natively expressing eGFP (green, top) and stained against GFP using a secondary antibody in the red spectrum (magenta, center) in $\mathrm{TH}$-cre, PRS $\times 8, D B H$-cre mice (from left to right). $\mathbf{b}$, Number of neurons detected by CellPose in the native eGFP (green, $x$-axis) and GFP-stained (red, $y$-axis) channels across animals. c, $\mathbf{d}$, Co-localization of neurons detected in the green and red channel, as defined by an overlap of cell masks $\geq 50 \%$ (based on cell masks detected in the GFP-stained channel). e, Distribution of normalized fluorescence in anti-GFP stained channel (red) as a function of the normalized fluorescence in the native eGFP channel (green) across cells. Fluorescence was averaged within each cell mask (detected in the GFP-stained channel), then binned into $50 \times 50$ equally spaced bins, and further smoothed by a $2 D 10 \times 10$ boxcar function. $f$, A nonlinear function was fitted to the normalized fluorescence values. The function is of the form $f_{\mathrm{R}}=\phi\left(\right.$ gain + bias $\left.\times \phi^{-1}\left(f_{\mathrm{G}}\right)\right)$, where $f_{\mathrm{R}}$ and $f_{\mathrm{G}}$ denotes the fluorescence in the red (GFP-stained) and green (native eGFP) channels respectively, and $\phi$ corresponds to the normal cumulative distribution function. The 'gain' parameter quantifies the overall change in fluorescence induced by the immunostaining, while the 'bias' parameter accounts for a possible asymmetry in the fluorescence increase depending on the native eGFP fluorescence. $g$, Estimated parameter values from the function presented in $\mathbf{f}$. The 'gain' parameter was larger than 0 , indicating an increase in fluorescence following GFP-staining. The 'bias' parameter was smaller than 1 , indicating that the increase in fluorescence was stronger for the dimmest cells in the native eGFP channel. In $\mathbf{b}, \mathbf{d}$, and $\mathbf{g}$, error bars denote the standard error of the mean (with $n=13$ ), while statistical significance is denoted by $\emptyset$ for non-significance, and $* / * *$ for $p<0.05 / 0.001$ respectively. 


\section{Ectopic expression of transgenes}

Besides differences observed with regard to specificity of neural transduction within the LC, we also observed ectopic GFP expression in different and widespread brain regions (Fig. 3). Again, we found substantial differences between the different model systems with respect to peri-LC transgene expression. The mildest ectopic expression was found in $D B H$-cre animals, where we only observed GFP expression in a small number of cells in the cerebellum $(<10$ neurons, 3/7 animals; Fig. S4a) and in the inferior colliculus ( $<10$ neurons, 1/7 animals; Fig. 3a). In PRS $\times 8$ animals, we found moderate GFP expression both in the vestibular nucleus ( $10-200$ neurons, 6/7 animals; Fig. S4b), and in the lateral parabrachial nucleus ( 10-100 neurons, 3/7 animals; Fig. 3b). Finally, in $\mathrm{TH}$-cre mice, we found substantial ectopic expression in the lateral parabrachial nucleus ( $10-500$ neurons, 7/7 mice; Fig. S4c), moderate expression in the inferior colliculus (10-100 neurons, 2/7 mice; Fig. 3c) and central gray $(<100$ neurons, $1 / 7$ mice; Fig. S4d) and mild expression in the cerebellum (<10 neurons, 1/7 mice; Fig. S4e).

\section{Success rates of $L C$ targeting}

In the original set of experiments, we observed complete absence of the GFP signal and, thus, transgene expression in one of the two LC of some animals, while expression was strong in the contralateral LC. Out of 5 bilaterally injected animals in each group, we observed unilateral transgene expression in 3/1/3 DBH/TH-/PRS $\times 8$-regulated mice respectively (Fig. S5a). In this first set of experiments, we used the same micropipette to inject the viral solution sequentially in each LC and were concerned that this approach might compromise the success rate of injections (e.g., due to inhomogeneous viral suspension). Therefore, we added two more mice to each group using a dedicated micropipette per LC. However, also here, we observed missing transgene expression in some cases with 0/2/1 $\mathrm{DBH}-/ \mathrm{TH}$-/PRS $\times 8$-regulated mice lacking expression in one of their LC respectively (Fig. S5a), precluding an explanation in terms of injection approach. Importantly, infusion of the viral suspension was also visually confirmed during each injection, and hence the absence of expression in some LC could not be attributed to clogged micropipettes.

Since conditional transgene expression was entirely absent in some LC despite ejection of the virus suspension in the target area, we aimed to estimate viral spread to test the possibility that LC were missed and nearby brain regions were infused instead. We injected $D B H$ - and $T H$-cre mice ( $n=3$, each) with a so-called 'cre-switch' construct which consists of a double-floxed open reading frame for tdTomato and inverted open reading frame for eGFP (DO-tdTomato-DIO-eGFP) ${ }^{34}$. Hence, cell type specific expression of eGFP in cre positive cells allows for characterization of the molecular specificity, while expression of tdTomato in cre negative cells allows for estimation of the general viral spread at the injection site (Fig. S5b). Expression of tdTomato in cre negative cells revealed a broad viral spread in most animals, reaching from the midline to the parabrachial nucleus in the mediolateral axis, and from the $4^{\text {th }}$ ventricle to the central gray in the dorsoventral axis (Fig. S5c). In these experiments, we observed one animal (out of 6) with unilateral expression. Importantly, in this case, the 
expression of tdTomato was also absent in the non-expressing hemisphere (Fig. S5c, topright), suggesting that most of non-expressing LC were not due to off-target injection in neighboring brain tissue, but rather failure of virus infection in general.

a
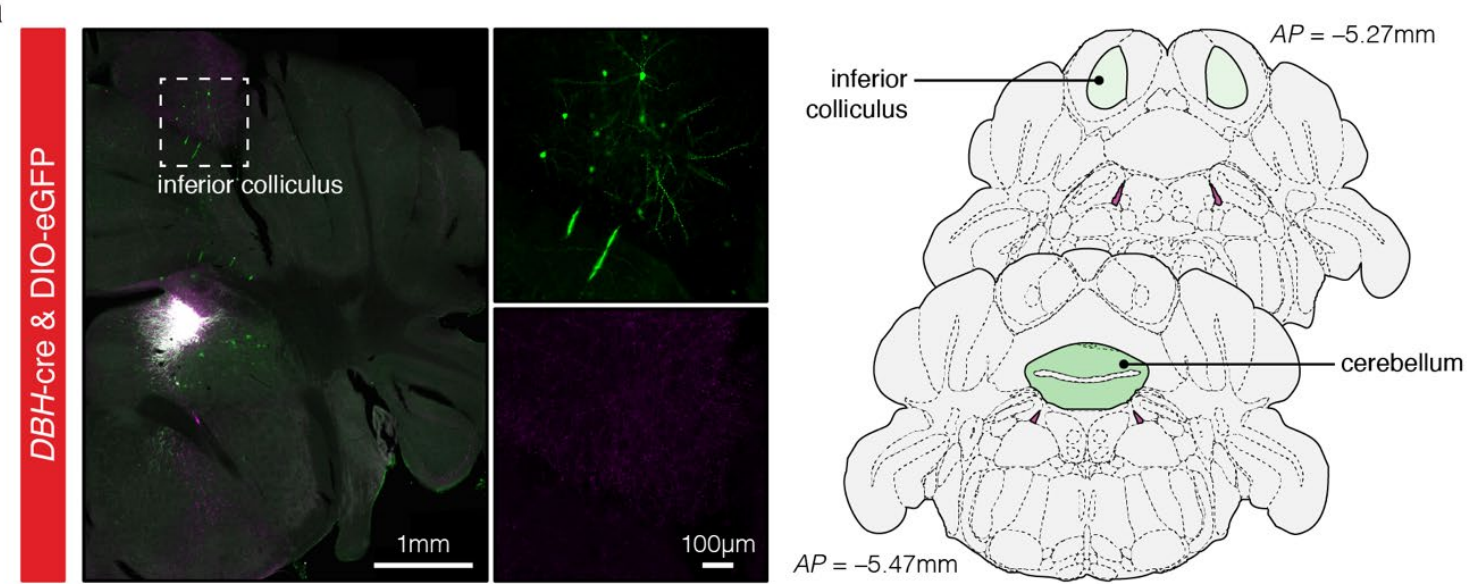

b
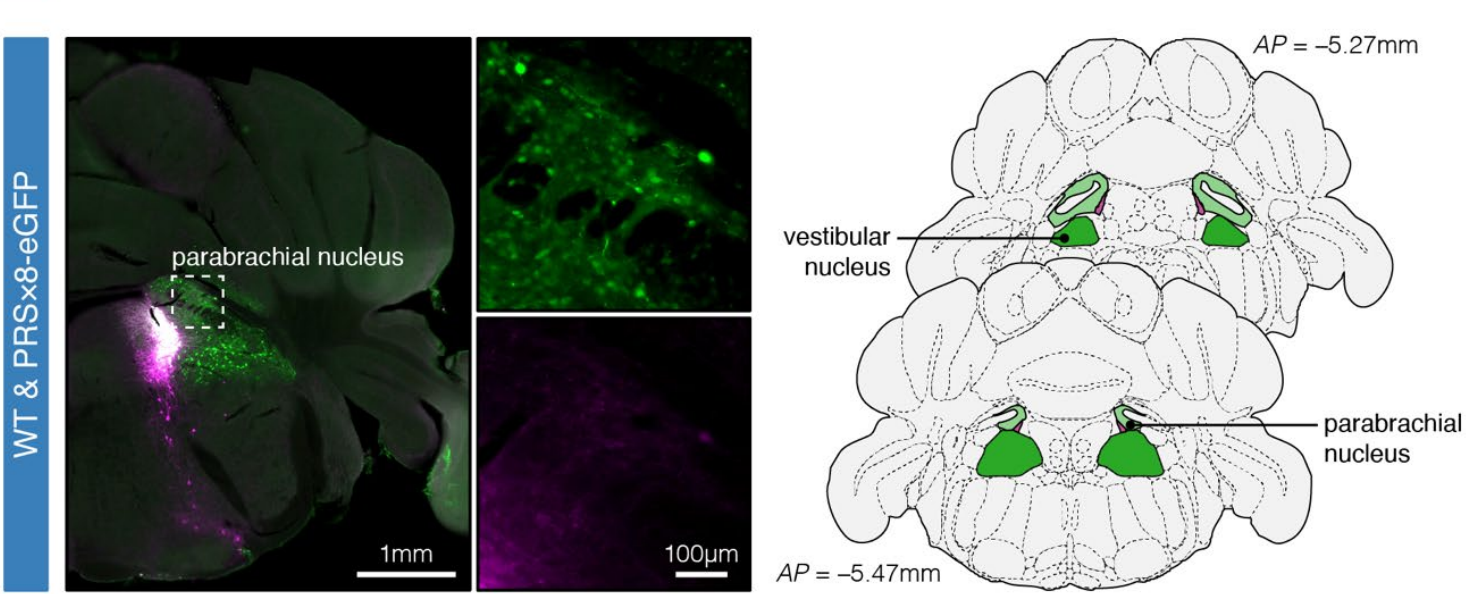

C

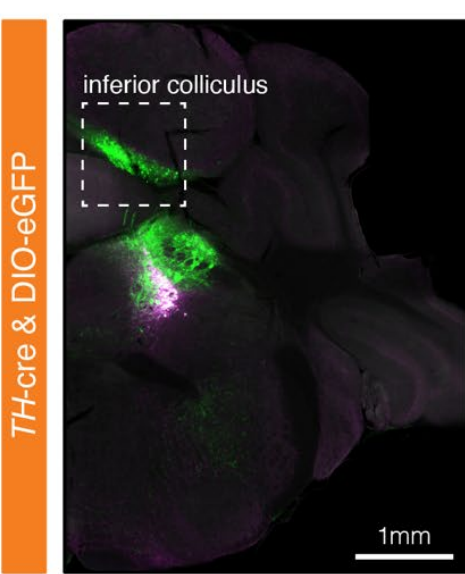

anti-TH anti-GFP

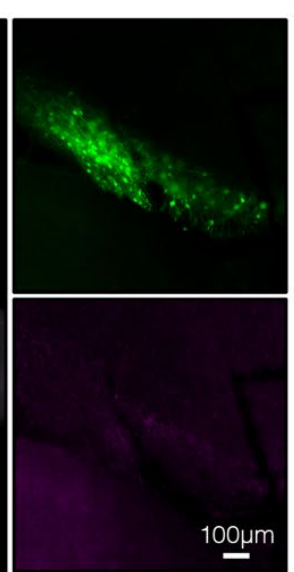

$100 \mu$

Figure 3 | Ectopic transgene expression across model systems, a-c, On the left panels; example brain slices showing ectopic expression in $\mathrm{DBH}$-/PRS $\times 8 / \mathrm{TH}$-cre model systems. Immuno-straining against TH and GFP is displayed in magenta and green respectively. On the right panels; summary of ectopic expression across all mice in $\mathrm{DBH}$-/PRS $\times 8 / \mathrm{TH}$-cre model systems. Brain regions are color-coded to reflect the number of mice which showed ectopic expression (at least 1 neuron) in the corresponding brain region. 
The overall success rate of injections in our study $(80 \%, 43 / 54$ LC) is in line with previous reports $(76-80 \%)^{35,36}$, which attributed the lack of transgene expression to off-target virus administration. However, given that our cre-switch experiments have revealed quite substantial viral spread, we assume that off-target administration into LC-proximal brain tissue would lead to similar viral spread, and consequently transgene delivery to the LC. Hence, we suggest that the lack of expression in some LC might result from off-target administration into the $4^{\text {th }}$ ventricle, which is in close proximity to the locus coeruleus.

\section{Discussion}

In this study, we performed a side-by-side comparison of three readily available model systems for virus-based genetic manipulation of the LC in mice, including LC transduction with floxed transgenes in $\mathrm{TH}$ - and $\mathrm{DBH}$-cre transgenic driver lines as well transgene expression under the control of a synthetic, NE-specific promoter (PRS $\times 8$ ). Despite their widespread use, comprehensive quantification of transgene expression is not always reported, and a direct comparison of these model systems is lacking.

Based on immuno-histological analysis, we report substantial differences, both in terms of expression efficacy and molecular specificity, between these model systems. While viral transduction in $D B H$-cre mice showed high efficacy and specificity, transduction driven by the PRS $\times 8$-promoter exhibited comparable efficacy, yet reduced specificity. Finally, transduction in $\mathrm{TH}$-cre mice showed both lower efficacy and specificity.

Furthermore, substantial ectopic transgene expression was observed in $\mathrm{TH}$-cre. At this point, it is important to note that TH-positive neurons in brain areas close to the LC, such as the parabrachial nucleus, have been reported ${ }^{37}$. While the ectopic expression in our study most likely was not localized to these TH-positive neurons (indicated by the absent TH staining), this population should be kept in mind as it may lead to wrong interpretations about the function of LC-NE neurons if LC-specificity in $\mathrm{TH}$-cre mice is taken for granted. Ectopic transgene expression was less pronounced in $\mathrm{PRS} \times 8$ mice and only very mild ectopic expression was observed in $\mathrm{DBH}$-cre mice.

Although a side-by-side comparison of virus-mediated LC transduction under comparable conditions (i.e., keeping titer, injection volumes, viral serotypes, etc. constant) has not yet been performed, several different studies report on the transduction efficacy and specificity individually for each of these model systems. In $\mathrm{DBH}$-cre mice, efficacy of viral transduction between $83 \%$ and $96 \%$ of $\mathrm{TH}$-immunoreactive neurons has been reported ${ }^{38-40}$, with a specificity of $95 \%{ }^{39}$. Using transgene expression mediated by the PRS $\times 8$ promoter, transduction efficacy of $83 \%$ of $\mathrm{TH}^{+}$neurons ${ }^{24}$ at a specificity of $100 \%{ }^{21,24}$ was reported in rats. In mice, a specificity of $95-98 \%$ was described, and high, yet not-quantified, efficacy was observed ${ }^{22,23}$. Furthermore, a more recent study by Stevens et $\mathrm{al}^{36}$ reported efficacies ranging from $\sim 50$ to $90 \%$, with specificities between $~ 60$ to $95 \%$ (dependent on the volume and titer 
of injected virus). This study reported the best results with a titer of $1 \times 10^{11} \mathrm{gc} / \mathrm{ml}(71-77 \%$ efficacy, $96 \%$ specificity). At higher titers $\left(1 \times 10^{12} \mathrm{gc} / \mathrm{ml}\right)$, specificity decreased to $60 \%$ while, at even higher titers, $\left(5-20 \times 10^{12} \mathrm{gc} / \mathrm{ml}\right)$ the efficacy decreased, and substantial toxicity was observed. The titer used in our study $\left(8 \times 10^{11} \mathrm{gc} / \mathrm{ml}\right)$ comes closest to the $1 \times 10^{12} \mathrm{gc} / \mathrm{ml}$ condition of Stevens et al., and comparable levels of efficacy (70-90\%) and specificity (60-80\%) were observed. Consequently, the specificity of $\mathrm{PRS} \times 8$ driven expression can likely be improved by using lower titers in the future. However, it is important to note that all these studies used PRS $\times 8$-controlled constructs in combination with recombinant adenoviruses ${ }^{21}$ or canine adenovirus 2 (CAV-2) 22-24,36, which have a different tropism than rAAV2/9 used in our study ${ }^{41}$. With respect to rAAVs, insufficient $P R S \times 8$ mediated transgene expression was reported with $\operatorname{rAAV} 2 / 7^{42}$ ( $20.6 \%$ efficacy), while robust expression was reported upon injection of $\mathrm{rAAV} 2 / 9^{35,43}$. These findings highlight the importance of viral serotype and careful titration of the viral titer when implementing the $\mathrm{PRS} \times 8$ promoter for genetic manipulations of the LC.

In $\mathrm{TH}$-cre mice, we show that cre-mediated expression only partially covers TH-positive LC neurons. Low efficacy and ectopic, cre-dependent transgene expression was also previously described in close proximity to the $\mathrm{LC}^{15}$, the substantia nigra ${ }^{15}$, the VTA ${ }^{44,45}$, and various brain regions not directly related to catecholaminergic signaling ${ }^{17,45,46}$. A detailed study by Lammel and colleagues ${ }^{45}$ reported specificities of 48 and $59 \%$ in the ventral tegmental area of TH-IREScre and $T H$-cre mice, respectively, which compares well to the specificity we observed in the locus coeruleus. Higher efficacy and specificity for $D B H$ - when compared to $T H$-cre mice was also reported for catecholaminergic neurons in the $A 1 / C 1$ area of the brainstem, but differences were not as pronounced as in our study ${ }^{47}$. Overall, our results are in line with past findings from various different studies investigating cre-dependent expression in the LC. Thus, our systematic comparison confirms the differences in transgene expression efficacy and specificity observed in $D B H$-cre mice as compared to $\mathrm{PRS} \times 8$ mediated transgene expression and $T H$-cre mice.

It is important to note that ectopic expression is by far not an issue limited to the $\mathrm{TH}$ promoter, but was also reported in a wide variety of model systems, including cholinergic and catecholaminergic cells in the retina ${ }^{48,49}$, dopaminergic cells in the dorsal raphe nucleus ${ }^{50}$ and the limbic system ${ }^{51}$, as well as somatostatin expressing interneurons ${ }^{52}$ and microglia ${ }^{53}$ in the cerebral cortex. Such ectopic expression might stem from the fact that promoters could only be weakly active, hence leading to low levels of native protein expression. Low expression levels of a direct reporter, such as a fluorophore, might not be a big concern, as the detected signal might be much weaker compared to true positive cells. However, even low levels of cre are sufficient to recombine floxed transgenes over time, leading to substantial expression of the transgene, and thereby to de-coupling of endogenous cre expression and cre-dependent transgene expression ${ }^{54,55}$. This explanation is in line with reports of homozygous Ai32 reporter mice where immunostainings revealed leaky channelrhodopsin-2 expression throughout the brain in the absence of cre, yet with an expression level which did not suffice to induce 
detectable photocurrents ${ }^{56}$. Other explanations for ectopic expression include legitimate, yet transient promoter expression during development ${ }^{17,26,27,45,55}$, changes in endogenous expression patterns resulting from transgene integration itself ${ }^{48}$, or recombination of cre in the germline ${ }^{55}$.

While the model systems we compared in this study show substantial differences in their specificity to target the locus coeruleus noradrenergic neurons, it is important to note that these different approaches have specific advantages. Clearly, the $D B H$-cre line is the most efficient system to target the locus coeruleus most completely and specifically. However, the $\mathrm{TH}$-cre mouse line - because it is based on a promoter upstream in the chain of catecholaminergic synthesis - in principle allows for molecular targeting of both noradrenergic and dopaminergic neurons in the same animals. As both $\mathrm{DBH}$-cre and PRS $\times 8$ mediated transgene expression enables reliable access to the LC-NE system, different aspects need to be considered when deciding which system to use. On the one hand, $D B H$-cre mice demonstrate higher specificity. Furthermore, the cre-lox system is widely established, and hence cre driver lines can readily be combined with off-the-shelf viral constructs which are readily available on commercial repositories. Lastly, driver lines can be crossed with various reporter lines, which allows for reproducible transgene expression without the need for stereotactic surgery. On the other hand, $\mathrm{PRS} \times 8$ enables a high degree of flexibility for manipulation and read-out of different molecularly defined circuits when combined with other cre lines than those related to the noradrenergic system. Also, the PRS $\times 8$-system can be implemented in species other than mice, where driver lines are not commonly available, as it has recently been successfully demonstrated in rats ${ }^{24}$. In this species, the only alternative driver line is a $\mathrm{TH}$-cre line (which - in contrast to mice - has been reported to be reliable both in terms of efficacy, and in terms of specificity) ${ }^{18,57}$. Finally, the PRS $\times 8$ system circumvents common pitfalls of cre driver lines, such as phenotypic alterations, which have been vastly reported for neuromodulatory mouse lines ${ }^{58-60}$. In sum, all available model systems offer unique features to elucidate different aspects of LC function. However, independently of the model system chosen, our study highlights the importance of rigorous post-mortem verification of viral transduction and the implementation of adequate controls for each system.

Caution must be taken when transferring our conclusions to different experimental settings. First, we have only relied on a single viral titer across model systems. While all experimental groups were injected with the same amount of virus, it has been shown that efficacy, specificity, and potential toxicity of PRS $\times 8$-mediated gene expression highly depend on the viral titer ${ }^{36}$. Hence, it might well be that higher efficacy and specificity of PRS $\times 8$ mediated gene expression can be achieved when optimizing the viral titer for a specific application. Furthermore, all our experiments were performed with rAAV2/9, and hence might not be translatable to different serotypes or even different viral families. Another limitation of our study concerns the choice of antibodies. For example, we have stained against tyrosine hydroxylase, where anti-DBH or anti-NET could have instead been chosen. 
However, all experimental groups were stained with the same antibody, and notably the outcome of our study did not favor the $\mathrm{TH}$-cre line. Finally, our study did not include the recently developed NET-cre driver line because it is not commercially available. It is worth noting, however, that efficient and specific transgene expression has already been reported for this line, albeit not with the same approach as used in our study ${ }^{20}$.

In conclusion, we present a quantitative side-by-side comparison of three widely used systems to genetically access the locus coeruleus in mice, which all have their own advantages and drawbacks. Our results can aid the design of future studies and the interpretation of past results obtained with the corresponding model system. Moreover, it will hopefully contribute to a deeper understanding of the heterogeneous functionality of the LC noradrenergic system in future studies.

\section{Material and Methods}

Animals. Experiments were performed on adult (2-6 months of age) $\mathrm{C} 57 \mathrm{BL} / 6 \mathrm{~J}-, \mathrm{TH}^{-15}$ and $D B H$-cre ${ }^{19}$ mice, housed in cages with nesting material with no more than 5 animals per cage at the animal facility of the Center for Molecular Neurobiology of the University Medical Center Hamburg-Eppendorf. Animals were kept in a $12 / 12 \mathrm{~h}$ light/dark cycle, at $22^{\circ} \mathrm{C}$ and $40 \%$ humidity, and access to water and food ad libitum. All experiments were performed in compliance with German law according to the directive 2010/63/EU of the European Parliament on the protection of animals used for scientific purposes. Protocols were approved by the Behörde für Gesundheit und Verbraucherschutz of the city of Hamburg.

Stereotactic injections. For surgical procedures, mice were either anesthetized with Midazolam/Medetomidine/Fentanyl (MMF; 5/0.5/0.05 mg/kg bodyweight in $\mathrm{NaCl}$, intraperitoneal), or with Isoflurane ( $5 \%$ for induction, $\sim 1.5 \%$ for maintenance) and sedation was confirmed by the paw withdrawal reflex. In the case of Isoflurane anesthesia, adequate analgesia was achieved with Buprenorphine $(0.1 \mathrm{mg} / \mathrm{kg}$ in $\mathrm{NaCl}$, intraperitoneal). After the head was shaved with a fine trimmer and sterilized with iodine (MundiPharma), mice were fixed with an auxiliary ear bar (EB-6, Narishige Scientific) in a stereotactic frame and positioned on a heating blanket to maintain body temperature. Eyes were covered with an eye ointment (Vidisic, Bausch + Lomb) to avoid drying. Subsequently, the scalp was opened ( $1 \mathrm{~cm}$ incision) and the skull was cleaned with $\mathrm{NaCl}$ (0.9\%; Braun) and a bone scraper (Fine Science Tools). Bregma and lambda were identified and stereotactically aligned. A small craniotomy was then performed above the locus coeruleus (AP: -5.4 ; $\mathrm{ML}: \pm 0.9$; DV: $-3.6 \mathrm{~mm}$ relative to bregma) using a dental drill (Foredom), and was constantly kept moist to prevent drying. The viral suspension was drawn into a borosilicate glass pipette (pulled from $0.2 \mathrm{~mm}$ glass capillaries with a PC-100 puller; Narishige) using negative air pressure. $0.3 \mu \mathrm{l}$ of titer-matched $\left(8 \times 10^{11}\right.$ $\mathrm{gc} / \mathrm{ml}$ ) viral suspension containing rAAV2/9 carrying plasmids encoding eGFP either in a credependent (double-floxed inverted reading-frame, DIO) manner under control of the CAG promoter (in the case of $\mathrm{TH}$ - and $\mathrm{DBH}$-cre animals) or unconditional under control of the PRS $\times 8$ 
promoter (in the case of $\mathrm{C} 57 \mathrm{BL} / 6 \mathrm{~J}$ mice) were bilaterally injected into the $\mathrm{LC}$ with an injection speed of $100 \mathrm{nl} / \mathrm{min}$ using a custom-made, air-pressure driven injection system. Injections of rAAV2/9-Ef1a-DO-DIO-tdTomato-eGFP $\left(2.3 \times 10^{13} \mathrm{gc} / \mathrm{ml}\right.$; kindly gifted from Bernardo Sabatini; AddGene plasmid \#37120 (4) $^{34}$ were performed in $T H$ - and $D B H$-cre animals to estimate the viral spread. The glass micropipette stayed in place for at least $5 \mathrm{~min}$ after finishing injection to avoid unnecessary viral spread and was then slowly withdrawn. The skin was closed with simple interrupted sutures and covered with iodine. Mice received analgesia (Carprofen; $4 \mathrm{mg} / \mathrm{kg}$ bodyweight, subcutaneous), and Atipamezole/Flumazenil/Buprenorphine (FAB) was administered (2.5/0.5/0.1 mg/kg bodyweight, intraperitoneal) to antagonize anesthesia in the case of MMF; Isoflurane anesthetized mice were simply removed from the Isoflurane. Mice were placed in a cage on a heating pad for recovery. Meloxicam was mixed with soft food for the following two days.

Tissue collection. For perfusions, mice were deeply anesthetized with Ketamine/Xylazine (180/24 mg/kg bodyweight, intraperitoneal), which was confirmed by the absence of the paw withdrawal reflex. Mice were fixed on a silicone pad and transcardially perfused with $\sim 50 \mathrm{ml}$ of phosphate buffered saline (PBS) to rinse out the blood and subsequently with $\sim 50 \mathrm{ml}$ of $4 \%$ paraformaldehyde (PFA; $\mathrm{pH} 7$; Carl Roth $\mathrm{GmbH}$ ) to fix the tissue. Brains were explanted and post-fixed in 4\% PFA for at least $24 \mathrm{~h}$. After fixation brains were embedded in 3\% agarose (Genaxxon Bioscience) and coronal slices of $\sim 50 \mu \mathrm{m}$ thickness were obtained using a vibratome (VT 1000S, Leica). Slices were stored in PBS at $4^{\circ} \mathrm{C}$ until they were stained and mounted on microscope slides.

Immunohistochemistry. Brain slices were blocked in 10\% normal goat serum (NGS) and $0.3 \%$ Triton $\mathrm{X}-100$ in PBS for $2 \mathrm{~h}$ at room temperature. Slices were then incubated for two days at $4^{\circ} \mathrm{C}$ in a carrier solution ( $2 \%$ NGS, $0.3 \%$ Triton X-100, in PBS) containing primary antibodies (rabbit against tyrosine hydroxylase, 1:1000, AB152, Merck Millipore; and chicken against green-fluorescent protein, 1:750, A10262, Invitrogen, Thermo Fisher Scientific) to allow for sufficient target protein-primary antibody interaction. Subsequently, slices were rinsed 3 times for $5 \mathrm{~min}$ in PBS. Following the washing steps, slices were incubated with secondary antibodies (Alexa Fluor 546 goat anti-rabbit, A11035, Invitrogen, Thermo Fisher Scientific; and Alexa Fluor 488 goat anti-chicken, A11039, Invitrogen, Thermo Fisher Scientific) diluted 1:1000 in the above-mentioned carrier solution over night at $4^{\circ} \mathrm{C}$. Washing steps were repeated as described before and slices were mounted on microscope slides using Fluoromount mounting medium (Serva, Germany).

Image acquisition. Confocal images of the LC were captured in a Z-stack ( $2 \mu \mathrm{m}$ step size) using an LSM 900 airyscan 2 microscope (Zeiss, Germany) with a Plan-APOCHROMAT 20X/0.8 objective, controlled by the ZEN 3.1 imaging software, with laser intensities and gain of the photomultiplier tubes adjusted to maximize signal and avoid saturation of images. Whole slices were imaged using a Zeiss AxioObserver epifluorescence microscope (Zeiss, Germany) with a Plan-APOCHROMAT 20X/0.8 objective, controlled by the ZEN 3.3 imaging 
software imaging software. Image ${ }^{61}$ was used to adjust image brightness, contrast, and create maximum intensity Z-projections.

Data analysis. Transgene expression was detected on the cellular level with the deeplearning-based algorithm CellPose ${ }^{30}$. Cell detection was cross-validated with quantification by two different experimenters, who counted stained cells with the cell counter function of ImageJ. Cell masks of segmented cells in the eGFP and TH channels exported from CellPose where then checked for overlap using custom-written MATLAB scripts. For quantification of transduction efficacy, co-expression was checked based on the cell masks in the TH channel, and masks which had an overlap of $\geq 50 \%$ with the masks in the GFP channel were defined as co-expressing. Similarly, co-expression was quantified based on the cell masks in the GFP channel, in order to quantify the specificity of transduction. The number of $\mathrm{TH}^{+}, \mathrm{GFP}^{+}$, and $\mathrm{TH}-$ GFP-co-expressing cells was subsequently summed up per animal, in order to avoid biasing the results based on (i) the different amount of $\mathrm{TH}^{+}$cells on individual slices and (ii) the total amount of slices taken from each animal (some slices had to be excluded due to tissue damage during the mounting process). Quantification of the effect of anti-GFP immunostaining on cell fluorescence was performed by fitting (least-squares) a binormal model of the form $f_{\mathrm{R}}=\phi\left(\right.$ gain + bias $\left.\times \phi^{-1}\left(f_{\mathrm{G}}\right)\right)+\varepsilon$ with $\varepsilon \sim N\left(0, \sigma^{2}\right)$; and where $f_{\mathrm{R}}$ and $f_{\mathrm{G}}$ denotes the fluorescence in the red (GFP-stained) and green (native eGFP) channels respectively, and $\phi$ corresponds to the normal cumulative distribution function. Comparisons between the two channels have been performed based on the cell masks detected in the GFP-stained channel, as we aimed to reveal additional cells upon GFP staining, which, by definition, are not detected in the native eGFP channel.

Statistics. One-way ANOVA was performed; when significant, post-hoc comparisons between groups were performed using Tukey's honestly significant difference criterion which controls for inflated risk of type-1 error (false positives) following multiple comparisons (using MATLAB's 'anova1' and 'multcompare' functions). All statistical tests were two-tailed, with a type-1 error risk set to $a=0.05$. In all figures, error bars correspond to standard error of the mean.

Author contributions: $A D$ and JSW conceived the project. CW performed surgical procedures with support of $A D$. CW performed histological work. $C W$ and $A D$ acquired data. $A D, M M$, and $C W$ analyzed data. $A D$ wrote the first draft of the manuscript, with support from all authors. MM prepared figures, with support from AD. JSW and AD supervised the work and JSW acquired funding.

Acknowledgements: The authors gratefully acknowledge Kathrin Sauter for plasmid cloning, Dr. Ingke Braren for production of viral constructs, and Stefan Schillemeit for expert technical support with histology. MM is supported by the Alexander von Humboldt Stiftung, and by the 
Fondation Bettencourt-Schueller. This work was supported by the German Research Foundation (DFG, SFB936-B8 to JSW).

Conflict of interest: The authors declare no conflict of interest.

\section{References}

1. Sturrock, R. R. \& Rao, K. A. A QUANTITATIVE HISTOLOGICAL STUDY OF NEURONAL LOSS FROM THE LOCUS COERULEUS OF AGEING MICE. Neuropathol. Appl. Neurobiol. 11, 55-60 (1985).

2. Poe, G. R. et al. Locus coeruleus: a new look at the blue spot. Nat. Rev. Neurosci. 21, 644659 (2020).

3. Chandler, D. J. et al. Redefining Noradrenergic Neuromodulation of Behavior: Impacts of a Modular Locus Coeruleus Architecture. J. Neurosci. Off. J. Soc. Neurosci. 39, 8239-8249 (2019).

4. Schwarz, L. A. \& Luo, L. Organization of the locus coeruleus-norepinephrine system. Curr. Biol. CB 25, R1051-R1056 (2015).

5. Szot, P. Common factors among Alzheimer's disease, Parkinson's disease, and epilepsy: possible role of the noradrenergic nervous system. Epilepsia 53 Suppl 1, 61-66 (2012).

6. Morris, L. S., McCall, J. G., Charney, D. S. \& Murrough, J. W. The role of the locus coeruleus in the generation of pathological anxiety. Brain Neurosci. Adv. 4, 2398212820930321 (2020).

7. McBurney-Lin, J., Lu, J., Zuo, Y. \& Yang, H. Locus coeruleus-norepinephrine modulation of sensory processing and perception: A focused review. Neurosci. Biobehav. Rev. 105, 190199 (2019).

8. Aston-Jones, G. \& Waterhouse, B. Locus coeruleus: From global projection system to adaptive regulation of behavior. Brain Res. 1645, 75-78 (2016).

9. Waterhouse, B. D. \& Navarra, R. L. The locus coeruleus-norepinephrine system and sensory signal processing: A historical review and current perspectives. Brain Res. 1709, 1-15 (2019).

10. Oe, Y. et al. Distinct temporal integration of noradrenaline signaling by astrocytic second messengers during vigilance. Nat. Commun. 11, 471 (2020).

11. Feng, J. et al. A Genetically Encoded Fluorescent Sensor for Rapid and Specific In Vivo Detection of Norepinephrine. Neuron 102, 745-761.e8 (2019).

12. Sun, F. et al. A Genetically Encoded Fluorescent Sensor Enables Rapid and Specific Detection of Dopamine in Flies, Fish, and Mice. Cell 174, 481-496.e19 (2018).

13. Patriarchi, T. et al. Ultrafast neuronal imaging of dopamine dynamics with designed genetically encoded sensors. Science 360, eaat4422 (2018).

14. Patriarchi, T. et al. An expanded palette of dopamine sensors for multiplex imaging in vivo. Nat. Methods 17, 1147-1155 (2020). 
15. Savitt, J. M., Jang, S. S., Mu, W., Dawson, V. L. \& Dawson, T. M. Bcl-x is required for proper development of the mouse substantia nigra. J. Neurosci. Off. J. Soc. Neurosci. 25, 67216728 (2005).

16. Gong, S. et al. Targeting Cre Recombinase to Specific Neuron Populations with Bacterial Artificial Chromosome Constructs. J. Neurosci. 27, 9817-9823 (2007).

17. Lindeberg, J. et al. Transgenic expression of Cre recombinase from the tyrosine hydroxylase locus: Transgenic Expression of Cre Recombinase. genesis 40, 67-73 (2004).

18. Witten, I. B. et al. Recombinase-Driver Rat Lines: Tools, Techniques, and Optogenetic Application to Dopamine-Mediated Reinforcement. Neuron 72, 721-733 (2011).

19. Tillage, R. P. et al. Elimination of galanin synthesis in noradrenergic neurons reduces galanin in select brain areas and promotes active coping behaviors. Brain Struct. Funct. 225, 785-803 (2020).

20. Wagatsuma, A. et al. Locus coeruleus input to hippocampal CA3 drives single-trial learning of a novel context. Proc. Natl. Acad. Sci. 115, E310-E316 (2018).

21. Hwang, D. Y., Carlezon, W. A., Isacson, O. \& Kim, K. S. A high-efficiency synthetic promoter that drives transgene expression selectively in noradrenergic neurons. Hum. Gene Ther. 12, 1731-1740 (2001).

22. Bacon, T. J., Pickering, A. E. \& Mellor, J. R. Noradrenaline Release from Locus Coeruleus Terminals in the Hippocampus Enhances Excitation-Spike Coupling in CA1 Pyramidal Neurons Via $\beta$-Adrenoceptors. Cereb. Cortex 30, 6135-6151 (2020).

23. Li, Y. et al. Retrograde optogenetic characterization of the pontospinal module of the locus coeruleus with a canine adenoviral vector. Brain Res. 1641, 274-290 (2016).

24. Hayat, H. et al. Locus coeruleus norepinephrine activity mediates sensory-evoked awakenings from sleep. Sci. Adv. 6, eaaz4232 (2020).

25. Schmidt-Supprian, M. \& Rajewsky, K. Vagaries of conditional gene targeting. Nat. Immunol. 8, 665-668 (2007).

26. Harno, E., Cottrell, E. C. \& White, A. Metabolic pitfalls of CNS Cre-based technology. Cell Metab. 18, 21-28 (2013).

27. Matthaei, K. I. Genetically manipulated mice: a powerful tool with unsuspected caveats. J. Physiol. 582, 481-488 (2007).

28. Sohal, V. S., Zhang, F., Yizhar, O. \& Deisseroth, K. Parvalbumin neurons and gamma rhythms enhance cortical circuit performance. Nature 459, 698-702 (2009).

29. Hitoshi, N., Ken-ichi, Y. \& Jun-ichi, M. Efficient selection for high-expression transfectants with a novel eukaryotic vector. Gene 108, 193-199 (1991).

30. Stringer, C., Wang, T., Michaelos, M. \& Pachitariu, M. Cellpose: a generalist algorithm for cellular segmentation. Nat. Methods 18, 100-106 (2021).

31. George Paxinos \& Keith B.J. Franklin. Paxinos and Franklin's the Mouse Brain in Stereotaxic Coordinates, Compact: The Coronal Plates and Diagrams. (2019).

32. Fischer, K. B., Collins, H. K. \& Callaway, E. M. Sources of off-target expression from recombinase-dependent AAV vectors and mitigation with cross-over insensitive ATG-out vectors. Proc. Natl. Acad. Sci. U. S. A. 201915974 (2019) doi:10.1073/pnas.1915974116. 
33. Botterill, J. J. et al. Off-Target Expression of Cre-Dependent Adeno-Associated Viruses in Wild-Type C57BL/6J Mice. eneuro 8, ENEURO.0363-21.2021 (2021).

34. Saunders, A., Johnson, C. A. \& Sabatini, B. L. Novel recombinant adeno-associated viruses for Cre activated and inactivated transgene expression in neurons. Front. Neural Circuits 6, (2012).

35. Rorabaugh, J. M. et al. Chemogenetic locus coeruleus activation restores reversal learning in a rat model of Alzheimer's disease. Brain 140, 3023-3038 (2017).

36. Stevens, L. et al. Optimized Parameters for Transducing the Locus Coeruleus Using Canine Adenovirus Type 2 (CAV2) Vector in Rats for Chemogenetic Modulation Research. Front. Neurosci. 15, 663337 (2021).

37. Vincent, S. R. Distributions of tyrosine hydroxylase-, dopamine-beta-hydroxylase-, and phenylethanolamine- $\mathrm{N}$-methyltransferase-immunoreactive neurons in the brain of the hamster (Mesocricetus auratus). J. Comp. Neurol. 268, 584-599 (1988).

38. Koga, K. et al. Ascending noradrenergic excitation from the locus coeruleus to the anterior cingulate cortex. Mol. Brain 13, 49 (2020).

39. Nomura, S. et al. Combined Optogenetic Approaches Reveal Quantitative Dynamics of Endogenous Noradrenergic Transmission in the Brain. iScience 23, 101710 (2020).

40. Nomura, S. et al. Noradrenalin and dopamine receptors both control CAMP-PKA signaling throughout the cerebral cortex. Front. Cell. Neurosci. 8, 247 (2014).

41. Lavoie, A. \& Liu, B. Canine Adenovirus 2: A Natural Choice for Brain Circuit Dissection. Front. Mol. Neurosci. 13, 9 (2020).

42. Stevens, L. et al. A Feasibility Study to Investigate Chemogenetic Modulation of the Locus Coeruleus by Means of Single Unit Activity. Front. Neurosci. 14, 162 (2020).

43. Vazey, E. M. \& Aston-Jones, G. Designer receptor manipulations reveal a role of the locus coeruleus noradrenergic system in isoflurane general anesthesia. Proc. Natl. Acad. Sci. 111, 3859-3864 (2014).

44. Kempadoo, K. A., Mosharov, E. V., Choi, S. J., Sulzer, D. \& Kandel, E. R. Dopamine release from the locus coeruleus to the dorsal hippocampus promotes spatial learning and memory. Proc. Natl. Acad. Sci. 113, 14835-14840 (2016).

45. Lammel, S. et al. Diversity of transgenic mouse models for selective targeting of midbrain dopamine neurons. Neuron 85, 429-438 (2015).

46. Min, N., Joh, T. H., Kim, K. S., Peng, C. \& Son, J. H. 5' Upstream DNA sequence of the rat tyrosine hydroxylase gene directs high-level and tissue-specific expression to catecholaminergic neurons in the central nervous system of transgenic mice. Mol. Brain Res. 27, 281-289 (1994).

47. Rukhadze, I. et al. Catecholaminergic $\mathrm{A} 1 / \mathrm{C} 1$ neurons contribute to the maintenance of upper airway muscle tone but may not participate in NREM sleep-related depression of these muscles. Respir. Physiol. Neurobiol. 244, 41-50 (2017).

48. Vuong, H. E., Pérez de Sevilla Müller, L., Hardi, C. N., McMahon, D. G. \& Brecha, N. C. Heterogeneous transgene expression in the retinas of the TH-RFP, TH-Cre, TH-BAC-Cre and DAT-Cre mouse lines. Neuroscience 307, 319-337 (2015). 
49. Gábriel, R., Erdélyi, F., Szabó, G., Lawrence, J. J. \& Wilhelm, M. Ectopic transgene expression in the retina of four transgenic mouse lines. Brain Struct. Funct. 221, 37293741 (2016).

50. Cardozo Pinto, D. F. et al. Characterization of transgenic mouse models targeting neuromodulatory systems reveals organizational principles of the dorsal raphe. Nat. Commun. 10, 4633 (2019).

51. Papathanou, M., Dumas, S., Pettersson, H., Olson, L. \& Wallén-Mackenzie, Å. Off-Target Effects in Transgenic Mice: Characterization of Dopamine Transporter (DAT)-Cre Transgenic Mouse Lines Exposes Multiple Non-Dopaminergic Neuronal Clusters Available for Selective Targeting within Limbic Neurocircuitry. eNeuro 6, (2019).

52. Hu, H., Cavendish, J. Z. \& Agmon, A. Not all that glitters is gold: off-target recombination in the somatostatin-IRES-Cre mouse line labels a subset of fast-spiking interneurons. Front. Neural Circuits 7, 195 (2013).

53. Zhang, B. et al. The specificity and role of microglia in epileptogenesis in mouse models of tuberous sclerosis complex. Epilepsia 59, 1796-1806 (2018).

54. Stuber, G. D., Stamatakis, A. M. \& Kantak, P. A. Considerations when using cre-driver rodent lines for studying ventral tegmental area circuitry. Neuron 85, 439-445 (2015).

55. Song, A. J. \& Palmiter, R. D. Detecting and Avoiding Problems When Using the Cre-lox System. Trends Genet. TIG 34, 333-340 (2018).

56. Prabhakar, A., Vujovic, D., Cui, L., Olson, W. \& Luo, W. Leaky expression of channelrhodopsin-2 (ChR2) in Ai32 mouse lines. PLOS ONE 14, e0213326 (2019).

57. Uematsu, A. et al. Modular organization of the brainstem noradrenaline system coordinates opposing learning states. Nat. Neurosci. 20, 1602-1611 (2017).

58. Chen, E. et al. Altered Baseline and Nicotine-Mediated Behavioral and Cholinergic Profiles in ChAT-Cre Mouse Lines. J. Neurosci. Off. J. Soc. Neurosci. 38, 2177-2188 (2018).

59. Costa, K. M., Schenkel, D. \& Roeper, J. Sex-dependent alterations in behavior, drug responses and dopamine transporter expression in heterozygous DAT-Cre mice. Sci. Rep. 11, 3334 (2021).

60. Chohan, M. O., Esses, S., Haft, J., Ahmari, S. E. \& Veenstra-VanderWeele, J. Altered baseline and amphetamine-mediated behavioral profiles in dopamine transporter Cre (DAT-Ires-Cre) mice compared to tyrosine hydroxylase Cre (TH-Cre) mice. Psychopharmacology (Berl.) 237, 3553-3568 (2020).

61. Schindelin, J. et al. Fiji: an open-source platform for biological-image analysis. Nat. Methods 9, 676-682 (2012). 


\section{Supplementary Material}

Supplementary table 1: Cre driver lines to target the noradrenergic system in mice

Supplementary Figure S1: Independence of histology quantification on the overlap threshold of cell masks

Supplementary Figure S2: Leakage of cre-dependent virus in wildtype mice

Supplementary Figure S3: Agreement between CellPose-based and human-based cell segmentation

Supplementary Figure S4: Ectopic transgene expression across model systems

Supplementary Figure S5: Verification of LC transduction and viral spread

Supplementary table 1: Cre driver lines to target the noradrenergic system in mice. Bold font indicates mouse lines used in this study.

\begin{tabular}{|c|c|c|c|c|}
\hline Strain name & Gene & Provider & Strain ID & Reference \\
\hline B6.Cg-Dbh ${ }^{\text {tm3.2(cre)Pjen } / J}$ & $\begin{array}{l}\text { Dopamine- } \beta- \\
\text { Hydroxylase }\end{array}$ & Jackson Laboratory & 033951 & Tillage et al. ${ }^{19}$ \\
\hline $\begin{array}{l}\text { Tg(Dbh- } \\
\text { cre)KH210Gsat/Mmucd }\end{array}$ & $\begin{array}{l}\text { Dopamine- } \beta- \\
\text { Hydroxylase }\end{array}$ & $\begin{array}{l}\text { Mutant Mouse Resource } \\
\& \text { Research Centers }\end{array}$ & $\begin{array}{l}036734- \\
\text { UCD }\end{array}$ & Gong et al. ${ }^{16}$ \\
\hline $\begin{array}{l}\mathrm{Tg}(\mathrm{Dbh}- \\
\mathrm{cre}) \mathrm{KH} 212 \mathrm{Gsat} / \mathrm{Mmucd}\end{array}$ & $\begin{array}{l}\text { Dopamine- } \beta \text { - } \\
\text { Hydroxylase }\end{array}$ & $\begin{array}{l}\text { Mutant Mouse Resource } \\
\& \text { Research Centers }\end{array}$ & $\begin{array}{l}032081- \\
\text { UCD }\end{array}$ & Gong et al. ${ }^{16}$ \\
\hline 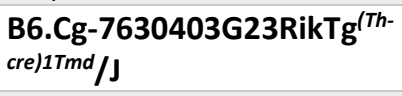 & $\begin{array}{l}\text { Tyrosine } \\
\text { Hydroxylase }\end{array}$ & Jackson Laboratory & 008601 & Savitt et al. ${ }^{15}$ \\
\hline $\begin{array}{l}\text { B6.FVB(Cg)Tg(Th- } \\
\text { cre)FI172Gsat/Mmucd }\end{array}$ & $\begin{array}{l}\text { Tyrosine } \\
\text { Hydroxylase }\end{array}$ & $\begin{array}{l}\text { Mutant Mouse Resource } \\
\text { \& Research Centers }\end{array}$ & $\begin{array}{l}031029- \\
\text { UCD }\end{array}$ & Gong et al. ${ }^{16}$ \\
\hline B6.129X1-Th ${ }^{\text {tm1(cre)Te } / \text { Kieg }}$ & $\begin{array}{l}\text { Tyrosine } \\
\text { Hydroxylase }\end{array}$ & $\begin{array}{l}\text { European Mouse Mutant } \\
\text { Archive }\end{array}$ & EM:00596 & $\begin{array}{l}\text { Lindeberg } \\
\text { al. }{ }^{17}\end{array}$ \\
\hline $\mathrm{Tg}($ Slc6a2-cre)\#Stl & $\begin{array}{l}\text { Norepinephrine } \\
\text { Transporter }\end{array}$ & - & - & $\begin{array}{l}\text { Wagatsuma et } \\
\text { al. }{ }^{20}\end{array}$ \\
\hline
\end{tabular}


a

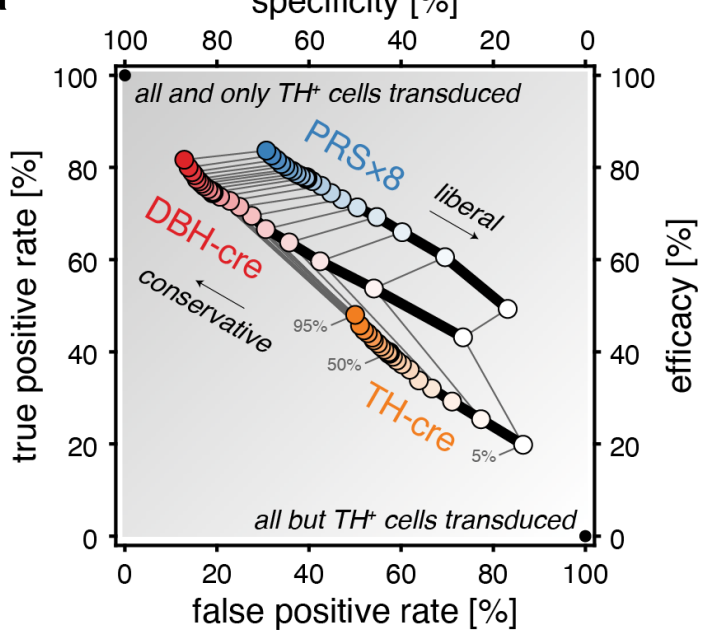

b

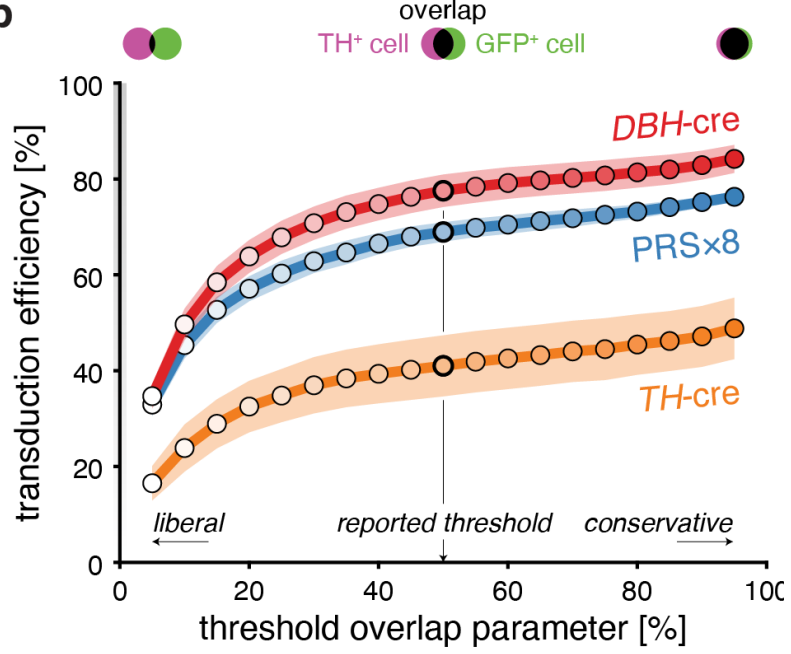

Figure S1 | Independence of histology quantification on the overlap threshold of cell masks. a, Efficacy and specificity from the 3 different model systems (as in Figure 1d) as a function of the threshold overlap parameter (light to dark colors; ranging from 5\% to $95 \%$, in steps of 5\%) which defines the minimal overlap between $\mathrm{GFP}^{+}$and $\mathrm{TH}^{+}$channels for a $\mathrm{TH}^{+}$cell to be also labeled as GFP ${ }^{+}$. b. Transduction efficiency (summarizing sensitivity and specificity in a single metric) from the 3 different model systems (as in Figure 1e) quantitatively varied as a function of the threshold overlap parameter. Importantly, conclusions were robust to this parameter choice as shown by the non-crossing of the curves (i.e., $T H$-cre $<\mathrm{PRS} \times 8<D B H$-cre). In the main text, we report values for a neutral threshold overlap parameter of $50 \%$. Shading areas denote the standard error of the mean (with $n=7$ ). 
bioRxiv preprint doi: https://doi org/10.1101/2022.01.22.477348; this version posted January 23, 2022. The copyright holder for this preprint (which was not certified by peer review) is the author/funder. All rights reserved. No reuse allowed without permission.

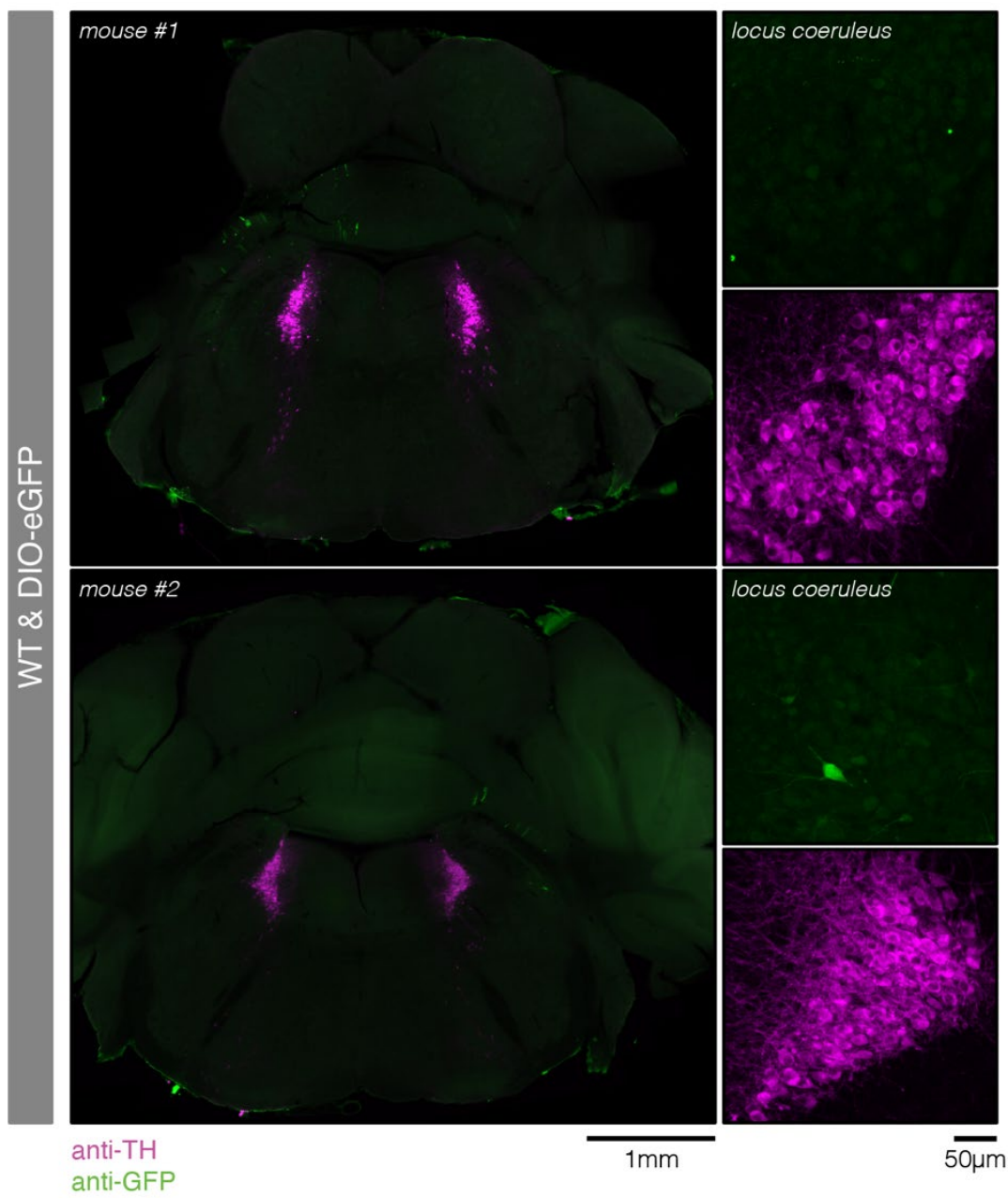

Figure S2 | Leakage of credependent virus in wildtype mice. Immunohistochemical staining against GFP (green) and TH (magenta) in two wildtype mice injected with rAAV2/9-DIO-eGFP. eGFP expression was observed in very few cells both within and outside of the $L C$. 
bioRxiv preprint doi: https://doi.org/10.1101/2022.01.22.477348; this version posted January 23, 2022. The copyright holder for this preprint (which was not certified by peer review) is the author/funder. All rights reserved. No reuse allowed without permission.

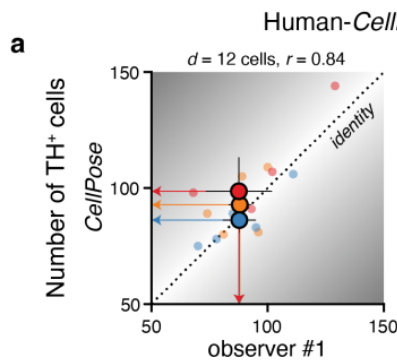

Human-CellPose agreement
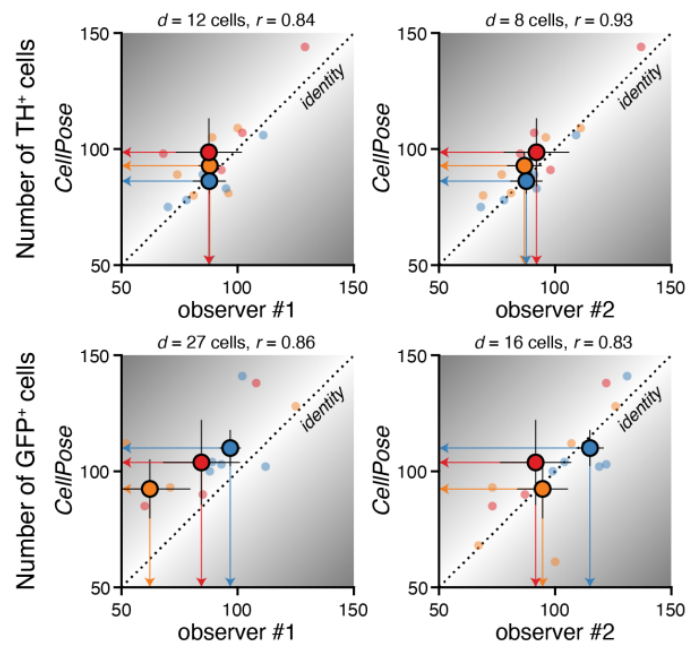

b
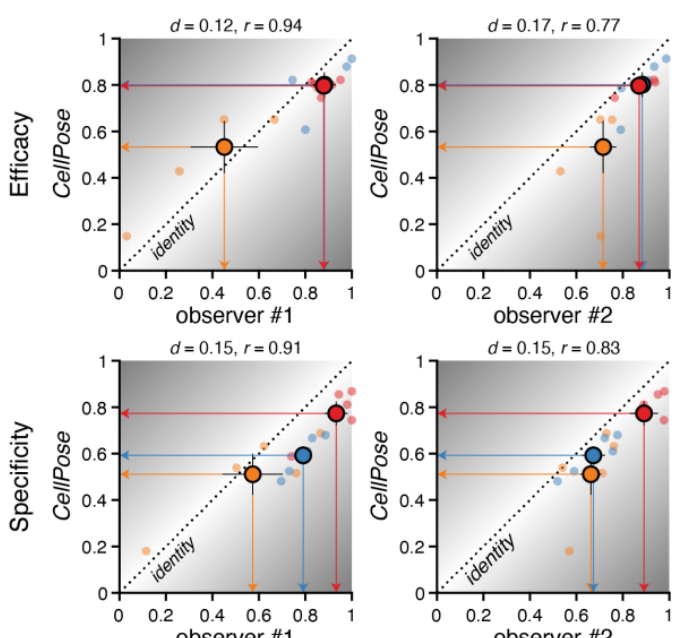

c
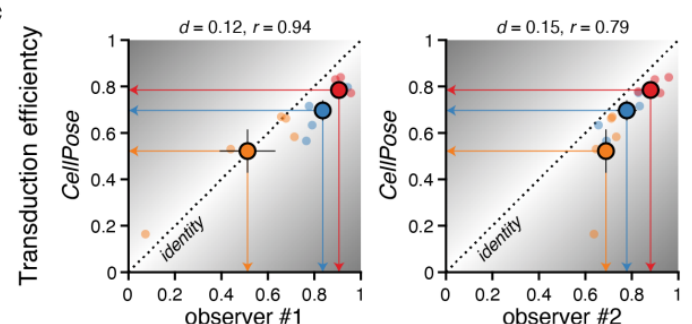

Inter-human agreement
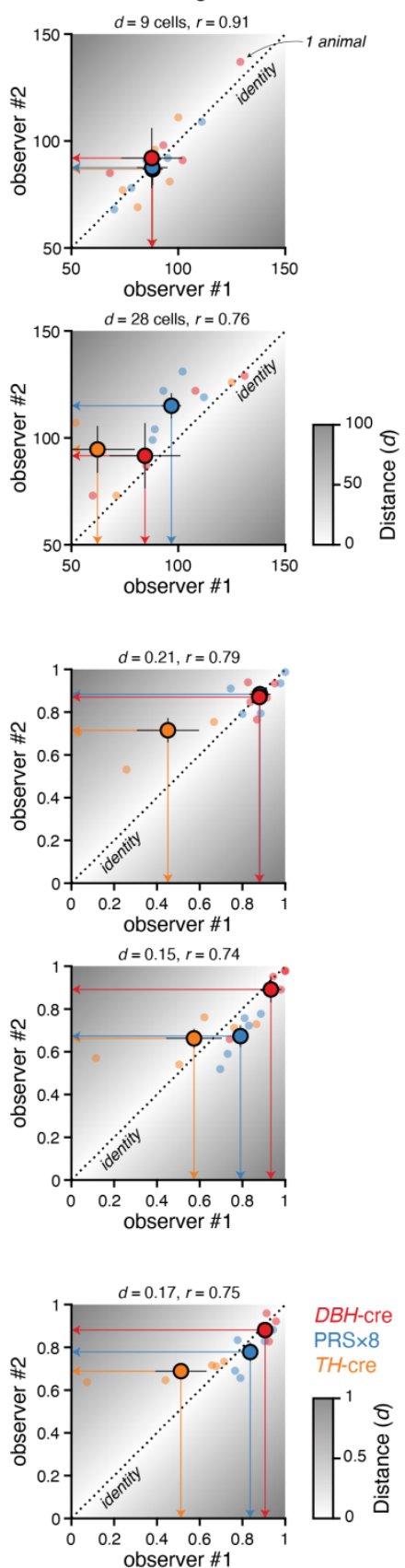

Figure S3 | Agreement between CellPose-based and human-based cell segmentation.

Correlation of cell counts; b, Efficacy \& specificity;

transduction efficiency between cells identified by CellPose vs. labeled by each of the two human observers (CW and AD; first 2 columns); and between the two human observers ( $3^{\text {rd }}$ column). A total of 5 slices of 5 different mice were used for each of the 3 model systems. Dashed lines indicate identity. In each case, two measures of agreement are reported: Pearson's correlation coefficient, denoted as $r$; and the distance between observers, denoted as $d=\sqrt{ } \sum_{1}\left(x_{i}-y_{i}\right)^{2}$ where $x$ and $y$ correspond to observers' reports (a) or derived quantities $(\mathbf{b}, \mathbf{c})$, and $i$ to the brain slice. Error bars denote the standard error of the mean (with $n=5$ ). 


\section{DBH-cre \& DIO-eGFP}
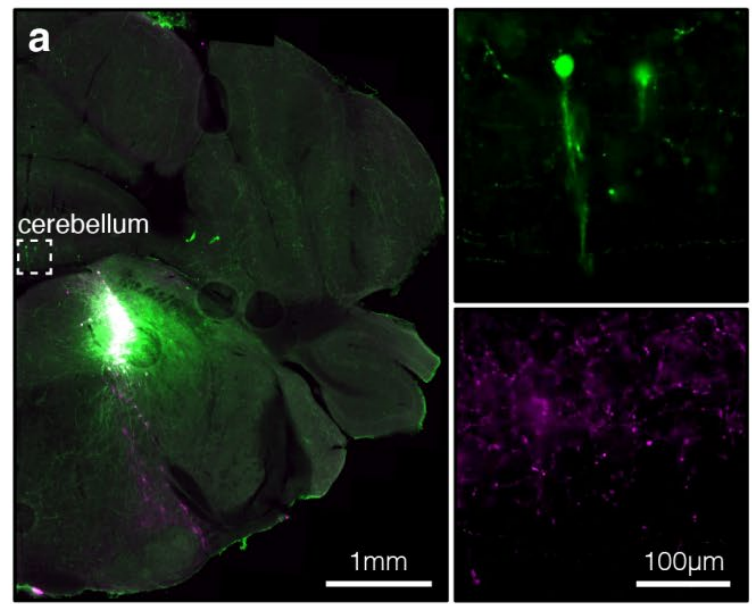

TH-cre \& DIO-eGFP
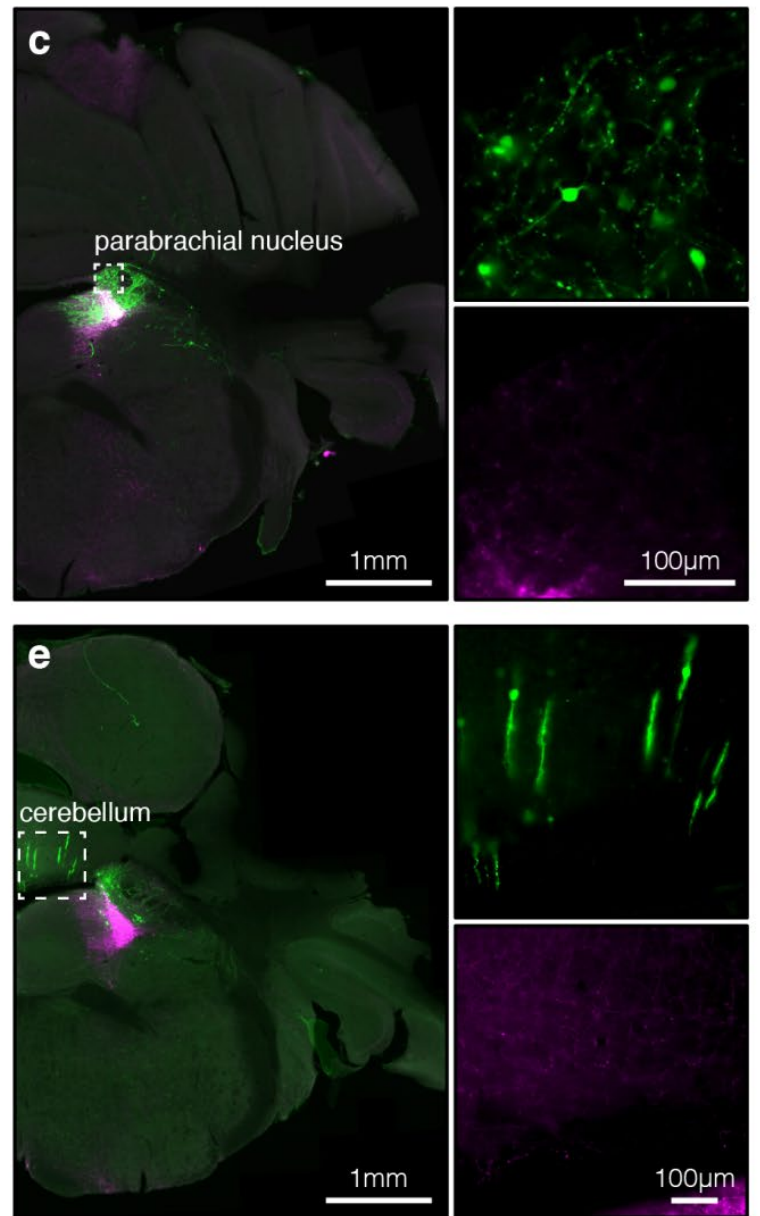

Figure S4 | Ectopic transgene expression across model systems. Example brain slices showing ectopic expression upon $D B H$-cre (a), PRS $\times 8$ (b), and $\mathrm{TH}$-cre (c-e) mediated transgene expression. Immuno-straining against TH and GFP is displayed in magenta and green respectively.

\section{WT \& PRS $\times 8-e G F P$}
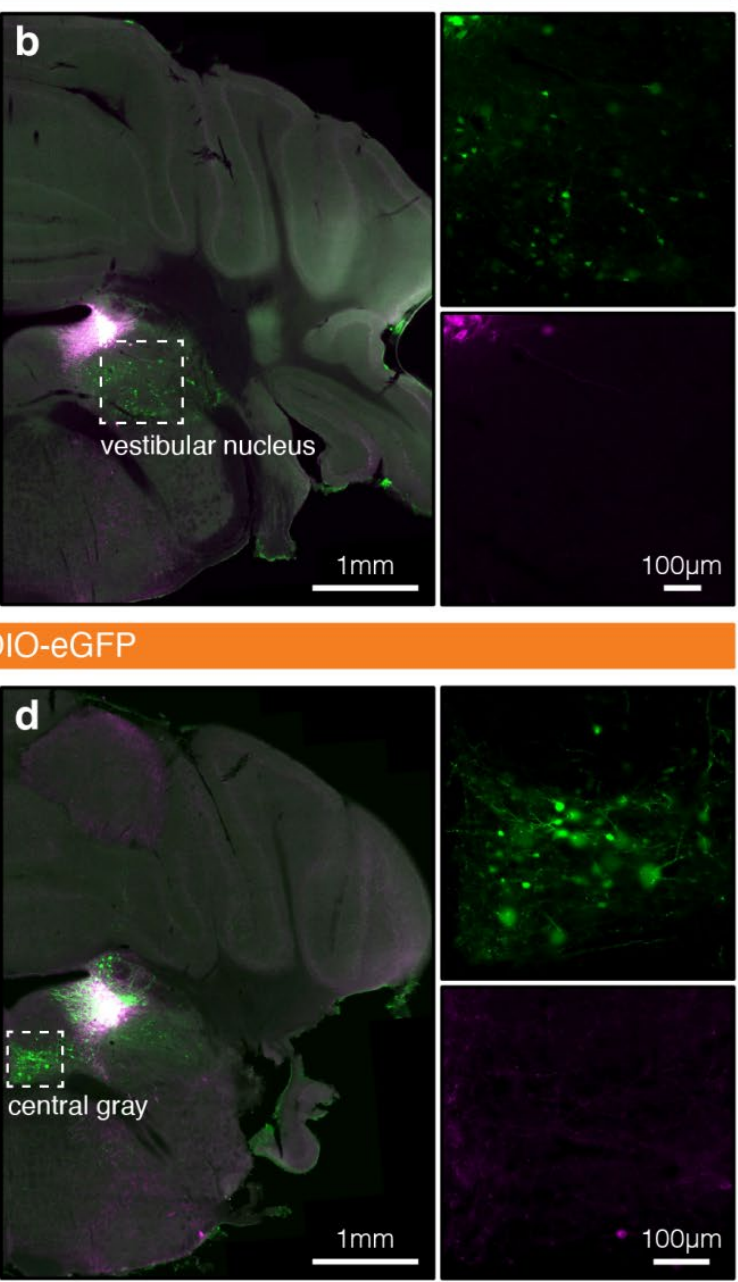

anti-TH

anti-GFP 
bioRxiv preprint doi: https://doi.org/10.1101/2022.01.22.477348; this version posted January 23, 2022. The copyright holder for this preprint (which was not certified by peer review) is the author/funder. All rights reserved. No reuse allowed without permission.

a
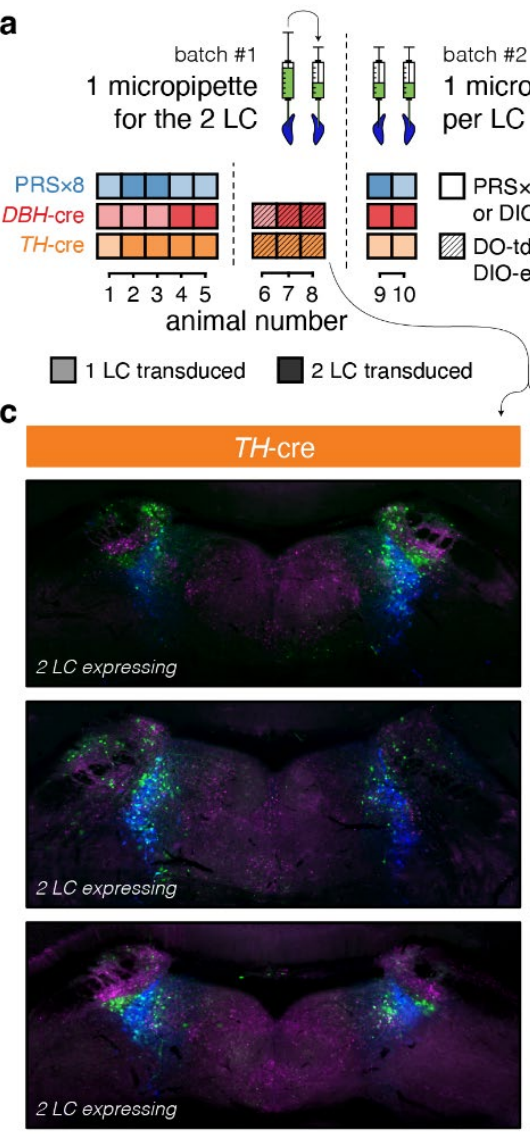

$1 \mathrm{~mm}$ b
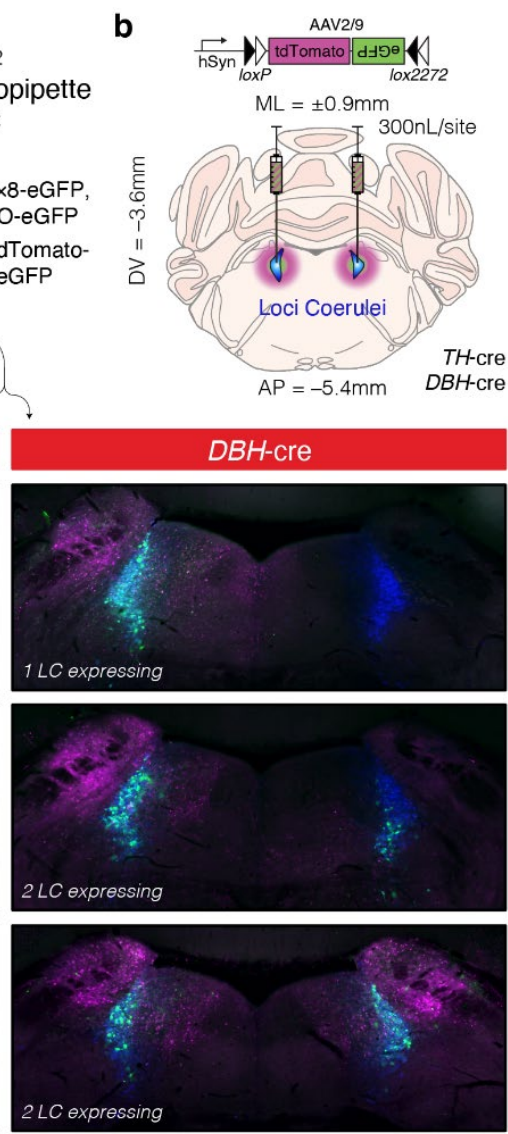

tdTomato

anti-GFP
Figure S5 | Verification of LC transduction and estimation of viral spread. a, Fraction of animals with 1 or 2 LC transduced. In the first surgery batch ( $n=5$ animals in each group), a single micropipette was loaded with enough virus $(2 \times 300 \mathrm{~nL})$ to infect both LC; the micropipette was then used to inject the virus sequentially in each LC. In the second surgery batch $(n=2$ animals in each group), one micropipette was used for each LC in order to control for possible differences in viral distribution, which might depend on the injection sequence and thereby explain failure in transducing one of the LC. In both batches, cases of single LC transduction were observed, suggesting they most likely followed from stereotactic targeting errors (with the pipette perhaps ending up in the $4^{\text {th }}$ ventricle). b, Viral spread was verified by injections of a so-called 'cre-switch' construct leading to eGFP expression in a cre-dependent manner and tdTomato expression in cre-negative cells $^{34}$. c, Transgene expression in $T H-$ and $D B H$-cre animals $(n=3$ animals each) shows conditional eGFP expression in cre-expressing cells (geen) and tdTomato expression in crenegative cells (magenta). LC cells are visualized by immunostaining against $\mathrm{TH}$ (dark blue). Importantly, in animals without eGFP expression, cre-negative cells around the LC also lacked the expression of tdTomato, suggesting absence of viral transduction in that hemisphere altogether. 